\title{
A Numerical Predictive Model for Conjugate Heat Transfer with Radiation
}

\author{
Marc-Paul Errera ${ }^{1, *}$, Rocco Moretti ${ }^{1}$, Julien Mayeur $^{1}$, Matteo Gelain $^{1}$ \\ ${ }^{1}$ DAAA, ONERA, Université Paris-Saclay, F-92322 Châtillon - France \\ and \\ Lionel Tessé ${ }^{2}$, Jean-Michel Lamet ${ }^{2}$, Emmanuel Laroche ${ }^{2}$ \\ ${ }^{2}$ DMPE, ONERA, Université de Toulouse, F-31055 Toulouse - France
}

\begin{abstract}
--- ABSTRACT ---
Taking into account radiation effects is a crucial part of the design and optimization of applications involving high temperatures. This paper outlines the development of a predictive coupling model for steady state conjugate heat transfer problems including radiative boundary conditions that models radiative exchanges between gray walls in a transparent medium. This canonical model is based on the Godunov-Ryabenkii normal mode analysis theory. The general expression of the amplification factor, the stability bounds and the optimal coefficients are provided. Moreover, a numerical Biot number including radiation effects that controls the stability of the model, is proposed. The destabilizing effect of radiation is highlighted and quantified. A specific test case is then presented to evaluate the consistency of this model. The numerical and physical parameters of this test case were specifically designed to target large fluid-structure interactions (ceramic material, high radiative coefficient). The numerical results fully comply with the theoretical results derived from the predictive model.
\end{abstract}

KEY WORDS: fluid-structure interaction, conjugate heat transfer, radiation, conduction, coupling

* Corresponding author: marc.errera@onera.fr +33(1) 46734315 


\section{A Numerical Predictive Model}

\section{Nomenclature}

\begin{tabular}{|c|c|}
\hline$a$ & thermal diffusivity $\left[\mathrm{m}^{2} \cdot \mathrm{s}^{-1}\right]$ \\
\hline$B i_{v}$ & numerical Biot number \\
\hline$D$ & Fourier number \\
\hline $\bar{D}$ & normalized Fourier number \\
\hline$F$ & Navier-Stokes flux \\
\hline$g$ & temporal amplification factor \\
\hline$h$ & heat transfer coefficient $\left[\mathrm{W} \cdot \mathrm{m}^{-2} \cdot \mathrm{K}^{-1}\right]$ \\
\hline$k$ & thermal conductivity $\left[\mathrm{W} \cdot \mathrm{m}^{-1} \cdot \mathrm{K}^{-1}\right]$ \\
\hline$K$ & thermal conductance $\left[\mathrm{W} \cdot \mathrm{m}^{-2} \cdot \mathrm{K}^{-1}\right]$ \\
\hline$N$ & number of beams of energy \\
\hline$q$ & heat flux $\left[\mathrm{W} \cdot \mathrm{m}^{-2}\right]$ \\
\hline$t$ & time $[\mathrm{s}]$ \\
\hline$T$ & temperature $[\mathrm{K}]$ \\
\hline$w_{f}$ & vector of fluid quantities \\
\hline$y+$ & $\begin{array}{l}\text { non-dimensional wall distance } \\
\text { complex variable }\end{array}$ \\
\hline$\Delta y$ & size $1^{\text {st }}$ cell $[\mathrm{m}]$ \\
\hline$\Delta t$ & time step $[\mathrm{s}]$ \\
\hline
\end{tabular}

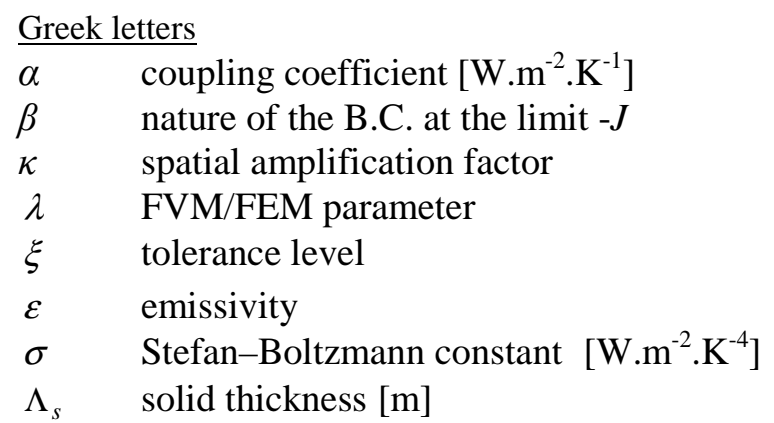

\section{$\underline{\text { Subscripts }}$}

$\begin{array}{ll}c & \text { coupled } \\ f & \text { fluid domain } \\ s & \text { solid domain } \\ r e f & \text { reference } \\ v & \text { numerical } \\ v_{f} & \text { inward unit normal to the fluid domain } \\ v_{s} & \text { outward unit normal to the solid domain }\end{array}$

\section{Superscripts}

$\begin{array}{ll}n & \text { temporal index } \\ \text { min } & \text { minimum } \\ \text { opt } & \text { optimal } \\ R & \text { radiative } \\ (\hat{\bullet}) & \text { unknown value }\end{array}$




\section{INTRODUCTION}

The coupling of heat transfer at a fluid-solid interface is usually known as conjugate heat transfer (CHT) [1][2]. CHT is used to analyze thermal interaction processes when the two modes of heat transfer - convection and conduction - are considered simultaneously. In recent years, the numerical analysis of CHT processes, without thermal radiation, has received extensive attention and many different numerical approaches for coupled problems have been proposed, on the basis of a normal mode analysis [3][4][5][6][7], the energy method [8], a matrix analysis [9], a steady-state approach [10], or a frequency-domain method [11]. In the context of "Large Eddy Simulation"-CHT problems, different acceleration techniques have been proposed [12][13][14][15].

However, radiation plays a key role since it is the dominant mode of heat transfer in participating media with applications involving high temperatures. Thus, thermal radiation coupled with convection and conduction can have extensive real-world applications when accurate heat transfer predictions are needed, such as reentry vehicles, turbomachinery, high temperature heat exchangers, combustion chambers, furnaces, etc.

Heat transfer involving simultaneously radiation, convection and conduction has been studied for a long time using approaches based on simple mathematical models. For instance, Goulard [16] studied the interaction of radiation and conduction in the Couette flow. Adrianov and Shorin [17] treated the case of coupled radiation and convection for a gray gas of a laminar flow in a tube. Flow between parallel plates was also considered and the effects of coupled radiation, conduction, and convection were treated by Einstein [18]. Desoto [19] developed an analytical procedure to investigate the interaction or coupling of radiation with the conduction and convection mechanisms in the entrance region of a black wall tube. In recent years, multidimensional approaches combining CFD, conjugate heat transfer and radiative heat transfer have been employed more frequently. These coupled calculations with boundary resolving meshes, produce results that provide a deep insight into CHT phenomena. For instance, Amaya et al. [20] [21] studied combustion applications on parallel computers. Duchaine et al. presented the partitioned coupling strategy [22] as well as the high performance computing criteria for coupled simulations [23]. A similar coupled approach has been adopted by Koren et al. [24] to predict the wall temperature distribution of a confined premixed swirling flame, using a dynamical coupling period. Mercier et al. [25] presented a coupling methodology to forecast the wall temperature distribution in a gas turbine combustor. Three different codes were used to deal with convection, conduction and radiation.

The most fundamental aspect in the coupling implementation is the choice of efficient conditions to connect the various physical phenomena. This is all the more important since these phenomena occur generally on a wide range of spatial and temporal scales. Thus, the interface conditions have a direct impact on the numerical properties of the coupling methodology. Our goal in this paper is to develop interface treatments of CHT problems with radiation on the basis of simple mathematical models, for a better handling of multidimensional codes in a coupled manner to solve multiphysics applications.

In CHT analysis, adaptive coupling coefficients have been highlighted and expressed for the first time by Errera and Chemin [26] for thermal fluid-structure interaction (FSI) in steady state problems and subsequent studies have been carried out [27][28][29]. For unsteady solid heat transfer, on the basis of the quasi-steady assumption, these coefficients are quite different and provided in [30]. These coefficients have been obtained from a 1D coupled model via a normal mode stability analysis based on the theory of Godunov-Ryabenkii [31][32][33][34][35]. The performance of these interface 


\section{A Numerical Predictive Model}

methods within the framework of Dirichlet-Robin boundary conditions were tested recently either in academic test cases [29] [30] or in an industrial furnace [36] and the relevance of the predictive model was fully confirmed.

The previous models were devoted to CHT analysis without radiation. The primary goal of the current paper is to perform an extensive stability analysis including radiative phenomena. A predictive 1D model is essential to explaining and quantifying the major issues pertaining to convergence and stability in coupled problems. This will be done by coupling three independent process models: the fluid model, the solid model, and radiation. The key issue in the coupling implementation is the interface condition. This condition has a direct impact on the numerical properties of the coupling methodology. Most of the interface numerical treatments in CHT problems are based on a Dirichlet transmission condition where temperature is prescribed on the fluid side and thus this condition will be adopted and illustrated in this paper.

The objective of this paper is to establish a numerical predictive model, stable and rapidly convergent for thermal coupling problems with radiation and based on a coupling approach in a partitioned strategy. Our goal is to provide the main parameters that control the coupling process and to express the coefficients that guarantee unconditional stability. Radiative exchanges between gray walls in transparent media are considered. Typical applications in the aerospace industry can be found in high temperature environments such as a jet engine combustor with hot external walls of the liner radiating on the internal wall of the casing.

This paper is structured as follows. First, the numerical model for CHT with radiation is outlined and the main parameters are provided and described (Section 2). Then, the numerical tools used in this study are briefly presented (Section 3). The following paragraph details the fluid-solid-radiation algorithm (Section 4). The numerical coupling procedure arising from the model is then applied to analyze the thermal interaction in a simple geometry with strong thermal fluid-solid interaction (Section 5). Finally, some concluding remarks are drawn. 


\section{COMPUTATIONAL MULTIPHYSICS}

\subsection{Partitioned approach for fluid-solid interaction}

\subsubsection{CHT Strategy in the fluid and in the solid domains}

The most efficient way to compute a numerical solution of the heat equation to a steady-state, provided the boundary conditions are time independent, is to consider a second order ordinary differential equation (Laplace's equation) and to solve for the temperature. A temporal algorithm would be an unnecessarily long procedure for the same result. As a consequence, only steady-state solid heat conduction may be considered. On the contrary, the Navier-Stokes equations are generally solved to steady-state by a temporal scheme. Thus, if these strategies perform well as single subsystems, they should be taken together and assembled in a multiphysics approach. However, solving Laplace's equation at each time step will change the solution too rapidly for stability to be maintained. In the current study, an optimal interface condition with remarkable stability properties is provided.

\subsubsection{Model problem}

Consider two finite domains with a common interface. The equations for the fluid domain are discretized using a finite volume method (FVM) and the heat conduction equation within the solid using a finite element method (FEM) as shown in Fig. 1. These domains are interactively solved to steady-state through a time-marching algorithm.

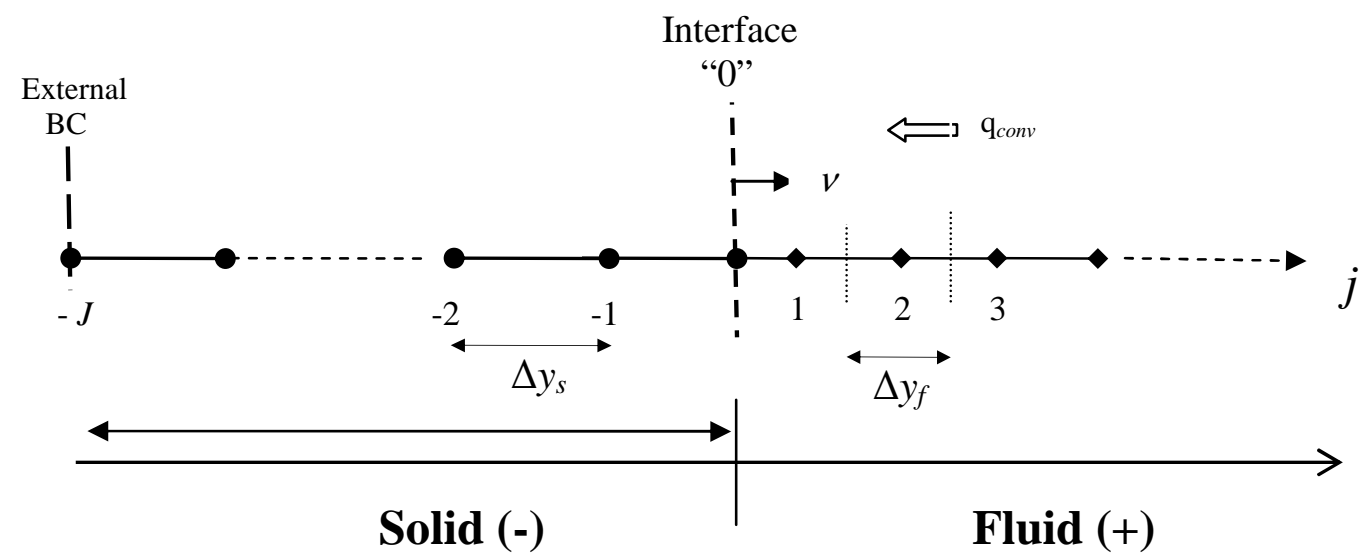

Figure 1 - Schematic of the fluid-solid interaction in the 1D model problem

\subsection{Fluid-solid interface treatment}

\subsubsection{Without radiation}

The fluid and solid domains are thermally coupled through a common interface and alternately integrated forward in time on the basis of a partitioned approach. We assume that the convection term is negligible in the first fluid cell. Simply stated, there is no velocity component normal to the fluidsolid (F-S) interface in 2D/3D flows. At this interface, a general Robin transmission condition is applied. In what follows, a common unit normal vector is adopted. This vector points towards the inward normal of the fluid domain (See Fig.1). The Robin boundary condition, also known as the mixed boundary condition or a boundary condition of the third kind is a linear relationship between 


\section{A Numerical Predictive Model}

the heat flux and the temperature [10][26][29]. With the convention signs just adopted, the Robin boundary condition on the fluid side becomes

$$
\hat{q}_{f}+\alpha_{s} \hat{T}_{f}=q_{s}+\alpha_{s} T_{s}
$$

and on the solid side

$$
\hat{q}_{s}-\alpha_{f} \hat{T}_{s}=q_{f}-\alpha_{f} T_{f}
$$

where $q$ is the heat flux, $T$ the temperature and $\left(\alpha_{f}, \alpha_{s}\right)$ are coupling coefficients. Quantities with the superscript $(\wedge)$ are the sought values. $q_{f}=q_{0+}=-K_{f} \partial T_{f} / \partial v$ is the normal heat flux where $v$ is the inward normal to the fluid domain. Similarly, $q_{s}=q_{0-}=-K_{s} \partial T_{s} / \partial v$, where $v$ is the outward normal to the solid domain. $K$ is the thermal conductance defined in the fluid finite volume model by

$$
K_{f}=\frac{k_{f}}{\lambda \Delta y_{f}}
$$

with $\lambda=1 / 2$ in a FVM and $\lambda=1$ in a FEM, $\Delta y_{f}$ being the size of the fluid cell adjacent to the wall. In a steady-state approach, the solid conductance is

$$
K_{s}=\frac{k_{s}}{\Lambda_{s}}
$$

where $\Lambda_{s}$ is the solid thickness. $k$ is the thermal conductivity.

Conditions (1)-(2) act to couple the two models and domains at the F-S interface. The coefficients $\left(\alpha_{f}, \alpha_{s}\right)$ are two adjustable parameters that control how heat is transferred across the F-S interface. They are the key stability factors of any numerical CHT procedure.

Note that, for simplicity, the Robin conditions (1) and (2) are expressed here with no temporal index. In Appendix A, these conditions are described in more details in a sequential approach and the temporal indices are indicated.

\subsubsection{With radiation}

In this study, only radiative heat exchanges between gray walls in transparent media are considered. Moreover, temperature differences in the computation domain are assumed sufficiently small to linearize radiative fluxes.

The general Robin interface condition on the fluid side becomes

$$
\hat{q}_{f}+\alpha_{s} \hat{T}_{f}+\hat{\Phi}_{f}^{R}=q_{s}+\alpha_{s} T_{s}
$$

and on the solid side

$$
\hat{q}_{s}-\alpha_{f} \hat{T}_{s}-\hat{\Phi}_{s}^{R}=q_{f}-\alpha_{f} T_{f}
$$

where $\Phi_{f}^{R}=\sigma \varepsilon\left(T_{f}^{4}-T_{r e f}^{4}\right)$ and $\Phi_{s}^{R}=\sigma \varepsilon\left(T_{s}^{4}-T_{r e f}^{4}\right)$ are the radiative heat fluxes on the fluid side and solid side respectively, with $\varepsilon$, the wall emissivity and $\sigma$ the Stefan-Boltzmann constant equal to $5.67 \times 10^{-8} \mathrm{~W} . \mathrm{m}^{-2} . \mathrm{K}^{-4}$. If the radiative fluxes can be linearized, the interface conditions (5) and (6) become

$$
\left\{\begin{array}{l}
\hat{q}_{f}+\left(\alpha_{s}+h_{f}^{R}\right) \hat{T}_{f}=q_{s}+\alpha_{s} T_{s}+h_{f}^{R} T_{r e f} \\
\hat{q}_{s}-\left(\alpha_{f}+h_{s}^{R}\right) \hat{T}_{s}=q_{f}-\alpha_{f} T_{f}-h_{s}^{R} T_{r e f}
\end{array}\right.
$$


with $\hat{\Phi}_{s}^{R}=h_{s}^{R}\left(\hat{T}_{s}-T_{\text {ref }}\right)$ and $\hat{\Phi}_{f}^{R}=h_{f}^{R}\left(\hat{T}_{f}-T_{\text {ref }}\right)$

and either: $h_{f}^{R}=\sigma \varepsilon\left(T_{f}+T_{\text {ref }}\right)\left(T_{f}^{2}+T_{\text {ref }}^{2}\right)$ and $h_{s}^{R}=\sigma \varepsilon\left(T_{s}+T_{\text {ref }}\right)\left(T_{s}^{2}+T_{\text {ref }}^{2}\right)$ (exact formulation) or: $h^{R}=4 \sigma \varepsilon T_{\text {ref }}^{3}$ (linearization).

At convergence, (7) becomes

$$
\left\{\begin{array}{l}
\left(\left[q_{f}+h_{f}^{R}\left(T_{f}-T_{r e f}\right)\right]-q_{s}\right)-\alpha_{s}\left(T_{s}-T_{f}\right)=0 \\
\left(\left[q_{s}-h_{s}^{R}\left(T_{s}-T_{r e f}\right)\right]-q_{f}\right)-\alpha_{f}\left(T_{s}-T_{f}\right)=0
\end{array}\right.
$$

It is easy to see that the convergence of temperature $\left(T_{f}=T_{s}\right)$ leads to the heat flux continuity $\left(q_{s}=q_{f}+h_{s}^{R}\left(T_{s}-T_{r e f}\right)=q_{f}+h_{f}^{R}\left(T_{f}-T_{r e f}\right)\right)$ across the interface, provided $\alpha_{f}+\alpha_{s} \neq 0 \quad\left(\right.$ and $\left.\frac{1}{\alpha_{f}}+\frac{1}{\alpha_{s}} \neq 0\right)$.

$\mathrm{Eq}$ (7) represents the most general form of interface conditions in a partitioned approach. This is a family of schemes depending on two coupling parameters. Two important aspects are to be considered at this stage. First, the Robin-Robin conditions constitute a very large family of potential interface conditions, not necessarily easy to set up since the joint use of two coefficients leads to a general and complicated relationship between both. Second, if continuity of flux and temperature is mathematically ensured at convergence, as just mentioned, it is far more complex in a discrete formulation and multiple solutions depending on the coupling coefficients can be obtained. This issue was largely discussed in [37] without radiation. With radiation, we have decided to strengthen the continuity, at every step of the process by imposing a single temperature value at the interface $T_{s}=T_{f}$ in the Robin equation of the solid domain. Thus, the generic system of Robin equations (7) on both sides of the interface becomes

$$
\left\{\begin{array}{l}
\hat{q}_{f}+\left(\alpha_{s}+h_{f}^{R}\right) \hat{T}_{f}=q_{s}+\alpha_{s} T_{s}+h_{f}^{R} T_{r e f} \\
\hat{q}_{s}-\alpha_{f} \hat{T}_{s}=q_{f}-\alpha_{f} T_{f}+h_{s}^{R}\left(T_{f}-T_{r e f}\right)
\end{array}\right.
$$

The current study will focus on a one-coefficient approach that is much easier to manage. Moreover, this approach inherently includes either the continuity of temperature with a Dirichlet condition or the continuity of heat flux with a Neumann condition. However, the Robin-Robin interface condition is an avenue that should be explored.

\subsection{Dirichlet-Robin interface treatment with radiation}

In the current paper, we will focus on the Dirichlet-Robin (D-R) interface approach obtained by imposing $\left(\alpha_{s}, \alpha_{f}\right)=\left(\infty, \alpha_{f}\right)$ in (9) which corresponds to a "perfect" conduction in the solid. When the solid conduction departs significantly from that assumption, in a solid ceramic material for instance, effective changes can be made to extend the scope of the D-R conditions so as to continue to retain this single interface treatment [37][38].

\subsubsection{Interface conditions}

In the D-R procedure, the temperature coming from the solid is applied on the fluid side and a "relaxed heat flux" is in turn used as a boundary condition for the solid

$$
\left\{\begin{array}{l}
\hat{T}_{f}=T_{s} \\
\hat{q}_{s}-\left(\alpha_{f}+h_{s}^{R}\right) \hat{T}_{s}=q_{f}-\alpha_{f} T_{f}-h_{s}^{R} T_{r e f}
\end{array}\right.
$$

At this stage, there are considerable benefits to note that the second equation in (10) is computed in the solid domain. It is then essential to express the temperature in the right-hand-side by a solid 


\section{A Numerical Predictive Model}

temperature only. Similarly, the radiative heat transfer coefficient can be expressed by a single temperature $\left(h_{s}^{R}=h_{f}^{R}=h^{R}\right)$. This leads to

$$
\left\{\begin{array}{l}
\hat{T}_{f}=T_{s} \\
\hat{q}_{s}-\left(\alpha_{f}+h^{R}\right) \hat{T}_{s}=q_{f}-\alpha_{f} T_{s}-h^{R} T_{r e f}
\end{array}\right.
$$

The latter expression considerably reduces the fluid-to-solid interpolation error at the interface and need only to transfer the interpolated heat flux to the solid. At convergence, it is easy to see that continuity of heat flux and temperature is obtained

$$
\left\{\begin{array}{l}
T_{f}=\tilde{T}_{s} \\
q_{s}=\tilde{q}_{f}+h^{R}\left(T_{s}-T_{r e f}\right)
\end{array}\right.
$$

where $\tilde{q}_{f}$ is the spatially interpolated heat flux from the fluid grid to the solid grid and similarly $\tilde{T}_{s}$ is the spatially interpolated solid temperature received at the fluid interface. Note that the solid temperature in the second equation contains no tilde since $T_{s}$ is directly available in the solid domain with no interpolation. Note also that the heat flux transferred to the solid (RHS in the second equation in (12)) does not need to be linearized since its exact value is known at each coupling step.

\subsubsection{Amplification factor}

Now, a normal mode solution for the case defined by the equations in the discrete model problem is applied according to the Godunov-Ryabenkii (G-R) stability analysis. The G-R stability analysis is very similar to the standard Fourier stability method except that the Fourier analysis ignores boundary conditions. However, these conditions may affect the stability [31].

The temporal amplification factor may be written as (see details in Appendices A and B):

$$
g\left(z, \alpha_{f}\right)=\frac{K_{f}}{\beta K_{s}+\alpha_{f}} \kappa_{f}\left(D_{f}, z\right)+\frac{\left(\alpha_{f}-K_{f}-h_{s}^{R}\right)}{\beta K_{s}+\alpha_{f}}
$$

where $\kappa_{f}$ is the spatial amplification factor (see Appendix B) that depends on the mesh Fourier number $D_{f}$ (defined in the next section) and on the complex variable $z$. In the denominator, $\beta$ is a parameter governing the nature of the $\mathrm{BC}$ at the outer limit of the solid $-J: \beta=1$, if a temperature is imposed and $\beta=0$ if a heat flux is imposed. (see [26] for details).

\subsection{Influence of radiation on stability}

Thus, it can be seen that $g(z)$ is a complicated non-linear equation in $z$. After the change of variable $z \rightarrow 1 / z$, the function $g$ becomes holomorphic on the open set $|z|<1$. As a result, on the basis of the maximum modulus principle in complex analysis, the maximum value of $|g|$ is achieved at the boundary. Using the maximum modulus principle in complex analysis, it can be demonstrated that the maximum of $\left|g\left(z, \alpha_{f}\right)\right|$ for $|z| \geq 1$ is located on the unit circle [26]. Thus

$$
\max _{|z| \geq 1}\left(g\left(z, \alpha_{f}\right) \mid\right)=\max _{|z|=1}\left(\left|g\left(z, \alpha_{f}\right)\right|\right)=\max _{|z|=1}\left(\left|g\left(\alpha_{f}\right)\right|\right)=\max (|g|)
$$

Actually, on the unit circle, it was shown [26] that the maximum switches from $z=-1$ to $z=+1$ as $\alpha_{f}$ increases and the amplification factor rebounds abruptly. At this point, $\max \{|g|\}$ attains its absolute minimum that is always located in the stable zone $|g|<1$. This intersection identifies the 


\section{A Numerical Predictive Model}

existence of a transition between two opposite zones. Switching from the first value to the second results in a sudden transition in the shape of the amplification factor composed of two half-lines with a singular point at the intersection of these two branches. The first branch is characterized by low values of $\alpha_{f}$ and the spatial amplification factor $\kappa_{f}\left(\alpha_{f}, D_{f}\right)$ is equal to a specific normalized Fourier number $\bar{D}_{f}$

$$
\bar{D}_{f}=\frac{D_{f}}{1+D_{f}+\sqrt{1+2 D_{f}}}
$$

where $D_{f}$ is the mesh Fourier number $D_{f}=a_{f} \Delta t / \Delta y_{f}^{2}$. The domain of $\bar{D}_{f}$ is $D_{f} \geq 0$ and the range is $0 \leq \bar{D}_{f}<1$. This branch, associated with $z=-1$, is time-dependent. For the specific value $\alpha_{f}=0$, the maximum of the amplification factor is easily obtained from (13)

$$
g(z, 0)=\frac{K_{f}\left(1-\bar{D}_{f}\right)+h^{R}}{K_{s}}
$$

The second branch is characterized by large values of $\alpha_{f}$ and $\kappa_{f}\left(\alpha_{f}, D_{f}\right)=1$. This branch, associated with $z=+1$, is time-independent.

In summary, the intersection of these two branches combines the advantages of the first two options, without suffering their disadvantages: the coupling process is fast and always stable. This is the reason why the conditions where this intersection occurs play a fundamental role in CHT.

Figure 2 shows the temporal amplification factor for three different radiative heat transfer coefficients $h^{R}=0(\varepsilon=0), h^{R}=311(\varepsilon=0.5), h^{R}=498(\varepsilon=0.8)$. All the other parameter values used to plot these curves are provided in Section 5.2. 


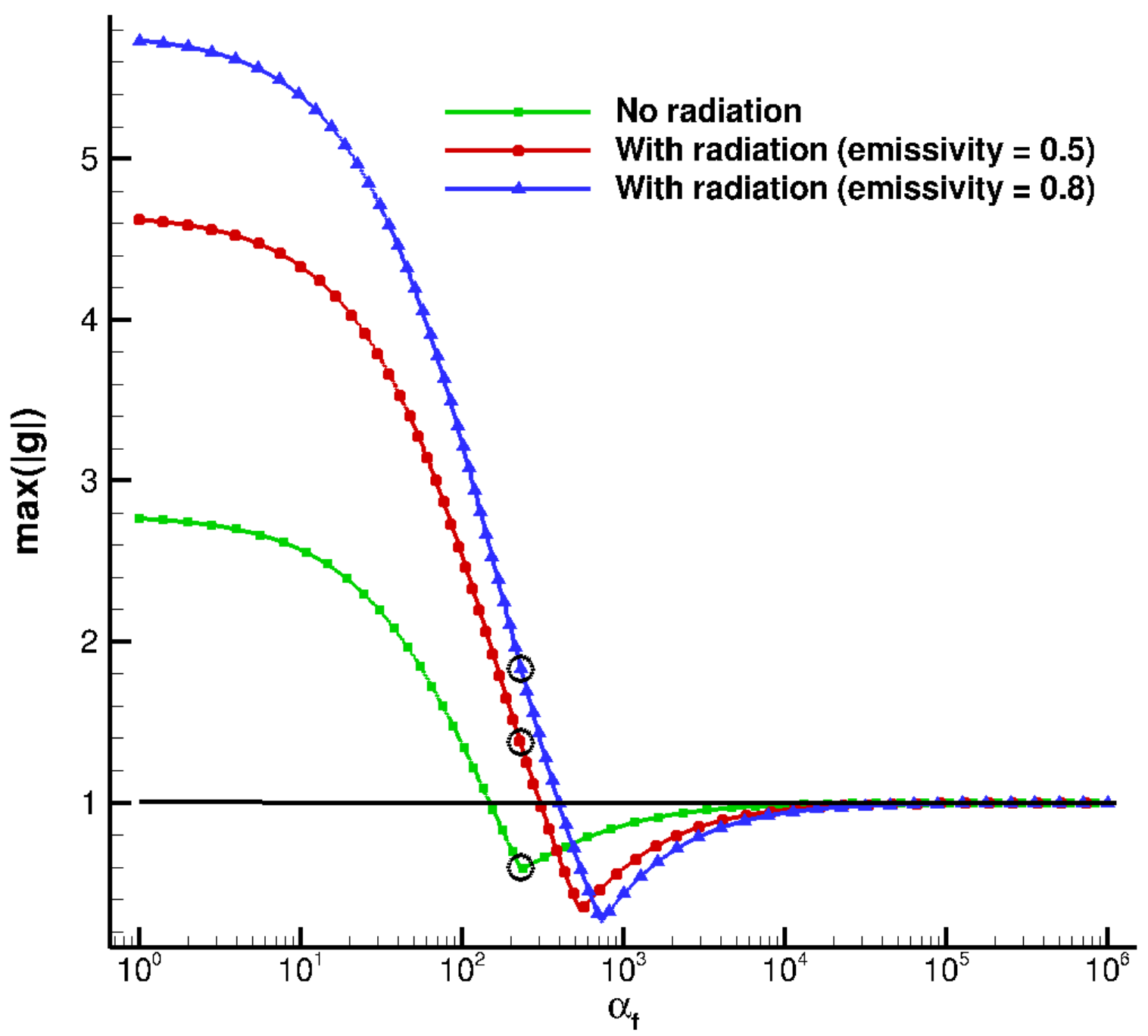

Figure 2 - Amplification factor of the model problem with and without radiation

It can be seen that radiation alters substantially the quantitative behavior of each curve. The $y$ intercept increases significantly (the exact value of the $y$-intercept is given by (16)), which has a negative impact on the stability. The same applies to the lower stability bound, $\max |g|=1$ which is shifted to the right. As a result, the optimal coefficients (single coefficients at the junction of the two branches) must also be increased to ensure stable computations with radiation.

With that in mind, Figure 2 brings to light a salient point. The optimal coefficient with no radiation, representing the absolute minimum of the curve, always less than unity, provides potentially unstable thermal computations with radiation $(\max |g|>1)$, represented by the small circles in Figure 2. The particular conditions necessary for this situation to arise are described in Section 5 and all the fundamental properties when radiation takes place are given in the next Section. 


\subsection{Fundamental properties of the thermal FSI with radiation}

\subsubsection{Optimal coefficient}

It is noteworthy that the modulus of the amplification factor goes through an absolute minimum, denoted $\alpha_{f}^{o p t}$ (without radiation) and $\alpha_{f-R}^{\text {opt }}$ (with radiation). In other words, the existence of a transition value for $\alpha_{f}$ can be identified: the shape of the curve of the amplification factor switches and rebounds as shown in Figure 2. These values can be conveniently represented using a shorthand notation (See [27][29] and Appendix B)

and

$$
\alpha_{f}^{o p t}=\frac{K_{f}}{2} \sqrt{\left(1+\frac{1}{D_{f}}\right)^{2}-1}-\frac{1}{D_{f}}=\frac{K_{f}}{2}\left(1-\bar{D}_{f}\right)
$$

$$
\alpha_{f-R}^{o p t}=\frac{K_{f}}{2}\left(1-\bar{D}_{f}\right)+h^{R}
$$

The subscript $f$ indicates that the coefficient is calculated in the fluid domain, and transmitted to the solid. Similarly, the subscript $f-R$ denotes that the coefficient is calculated in the fluid including radiative effects, and then sent to the solid. Eq.(18) highlights the need of an appropriate definition of a coupling coefficient for radiation. The multiphysics coupling strategy will be defined in Section 4 .

\subsubsection{Stability bound}

The stability bound $\alpha_{f-R}^{\min }$ is calculated from the condition $\left|g\left(z, \alpha_{f-R}^{\min }\right)\right|=1$. After applying a few basic calculus transformations (see Appendix B), the following expression is obtained

$$
\alpha_{f-R}^{\min }=\frac{K_{f}}{2}\left(1-\bar{D}_{f}\right)+\frac{h^{R}}{2}-\frac{\beta K_{s}}{2}
$$

It is a lower stability bound, i.e., the lowest value ensuring stability. Put another way, all coefficients lower than $\alpha_{f-R}^{\min }$ lead to a temporal factor greater than unity and thus theoretically to an unstable behavior.

\subsubsection{Numerical Biot number}

The stability bound given by Eq. (19) can be re-written

$$
\alpha_{f-R}^{\min }=\frac{\beta K_{s}}{2}\left(B i_{v}-1\right)
$$

with

$$
B i_{v}=\frac{K_{f}}{\beta K_{s}}\left(1-\bar{D}_{f}\right)+\frac{h^{R}}{\beta K_{s}}
$$

$B i_{v}$ may be regarded as a local numerical Biot number involved in a transient CFD calculation. Let us recall that the conventional Biot number needs a heat transfer coefficient. This new dimensionless number, naturally introduced by Eq. (20), is defined at any time in the transient state of a CHT computation and is a result of the balance between the transient fluid and the solid domain. This demonstrates how stability depends mainly on the ratio of thermal resistances, but also on the dynamics of the transient fluid system via the temporal term $\bar{D}_{f}$.

\subsubsection{Weak and strong thermal interaction}

As the coupling coefficient is always positive, two zones are clearly identified: 


\section{A Numerical Predictive Model}

(1) $B i_{v}<1$ : the coupling process is stable $\forall \alpha_{f-R} \geq 0$

(2) $B i_{v}>1$ : the coupling procedure exhibits a stability bound $\alpha_{f-R}^{\min }$.

From that, we naturally introduce the "strength" of the thermal FSI with radiation:

- Weak interaction: $B i_{v}<1$. If this condition holds, the "transient" thermal resistance of the fluid domain at the shared interface is greater than the resistance offered by the whole solid domain. Note that this resistance is strongly influenced by the time step. A Dirichlet interface condition on the fluid side is therefore appropriate.

- Moderate interaction: $B i_{v}>1$. This means either that the solid thermal gradients are not negligible or that the thermal fluid conductance is larger than that of the solid.

- Strong interaction: $B i_{v}>>1$. In this case, a Dirichlet interface condition imposed on the fluid does not provide the most efficient solution. However, it should be underlined that there is a powerful argument in favor of the Dirichlet condition. Indeed, the term $\left(1-\bar{D}_{f}\right)$ may become small by increasing the coupling period and the strength of the thermal FSI can therefore be significantly reduced.

\subsection{Impact of radiation on stability}

\subsubsection{Destabilizing effects}

Equation (21) can offer precious help to assess the potential influences of each term. It can be seen that all factors contributing to the increase of $\alpha_{f}^{\min }$ have a negative impact on stability and the reverse is also true. On this basis, it is easy to understand and quantify the impact of each term in the stability coupling with radiation:

- $\frac{K_{f}}{2}\left(1-\bar{D}_{f}\right)$ : increasing the fluid conductance, $K_{f}$, has a negative effect on stability (low Prandtl number, increase of the fluid viscosity, small fluid cell, ...). However, it should be kept in mind that the fluid conductance is paired with the temporal term $\left(1-\bar{D}_{f}\right)$. First, let's note that

$$
\frac{K_{f}}{2}\left(1-\bar{D}_{f}\right)=\frac{K_{f}}{1+\sqrt{1+2 D_{f}}}
$$

The Fourier number, $D_{f}$, is thus capable of stabilizing any CHT computation in two ways. First, even if $K_{f} \rightarrow \infty$ when $\Delta y_{f} \rightarrow 0$, it can be easily shown that (22) tends toward a finite value (see [37] for more details) given by

$$
\lim _{\Delta y_{f} \rightarrow 0} \frac{K_{f}}{1+\sqrt{1+2 D_{f}}}=\frac{2 k_{f}}{\sqrt{2 a_{f} \Delta t}}
$$

This shows that even if the term in Eq.23 is increasing as $\Delta y_{f}$ decreases, it reaches a limit. Thus, accurate conjugate heat transfer solutions that require high-resolution CFD meshes can be obtained with no instabilities. Second, the dynamic conductance decreases as $\Delta t^{-1 / 2}$ and thus the time step is capable of stabilizing most CHT calculations. This relationship gives us a better understanding of instabilities due to frequent couplings. An alternative interpretation is that the optimal coefficient is 


\section{A Numerical Predictive Model}

inversely proportional to the heat penetration depth $\sqrt{2 a_{f} \Delta t}$ showing that a small diffusion of heat during $\Delta t$ has a destabilizing effect.

- $\frac{h^{R}}{2}$ : this radiative term is always destabilizing and there is no temporal counterpart, as previously, to reduce this effect.

- $\quad \frac{\beta K_{s}}{2}$ : when $K_{s}=k_{s} / \Lambda_{s}$ is large, this contributes to strengthening the coupling process since this solid term reduces the value of $\alpha_{f}^{\min }$. This contribution is all the more important when the solid is a good thermal conductor (large conductivity $k_{s}$ ) or when the solid thickness $\Lambda_{s}$ is small. Conversely, in the case of a low contribution of the solid term (ceramics for instance or large solid thickness) stability issues may arise.

At this point, it is legitimate to wonder at which theoretical value of $h^{R}$, the thermal coupling with radiation becomes unstable if nothing is done to take it into account. Let us consider that the coefficient $\alpha_{f}^{o p t}$ (without radiation) is implemented. From the definition of $|g|$, we obtain

$$
\left|g\left(\alpha_{f}^{o p t}\right)\right|=\frac{-K_{f} \cdot \bar{D}_{f}-\alpha_{f}^{o p t}+K_{f}+h^{R}}{\beta K_{s}+\alpha_{f}^{o p t}}
$$

Instability is likely to occur when $|g|>1$, that easily reduces to the following condition

$$
h^{R}>\beta . K_{s}
$$

So, when the radiative heat transfer coefficient dominates the solid thermal conductance, the coupling process becomes theoretically unstable.

Note that the solid conductance is maximum for $\beta=1$, corresponding to the Dirichlet condition at the outer boundary, value adopted from now on.

\subsubsection{Stabilizing effects}

Consider yet again the amplification factor (Eq. 13). We see that its value for the optimal coefficient with radiation is given by

$$
\left|g\left(\alpha_{f-R}^{o p t}\right)\right|=\frac{\alpha_{f}^{o p t}}{K_{s}+\alpha_{f-R}^{o p t}}
$$

This shows that $\left|g\left(\alpha_{f-R}^{\text {opt }}\right)\right|<\left|g\left(\alpha_{f}^{\text {opt }}\right)\right|$. This effect can easily be seen in Figure 2, where the absolute minima of the curves with $\varepsilon>0$ are located slightly below that of the curves without radiation $\varepsilon=0$. Although the differences are small, a slightly faster convergence speed is expected, as long as the optimal coefficients are employed with and without radiation. 


\section{A Numerical Predictive Model}

\subsection{Summary of the theoretical results}

The main results of the stability analysis are summarized in Table 1. This table shows the behavior of the amplification factor ( $1^{\text {st }}$ column) as a function of the coupling coefficient $\alpha_{f-R}$ ( $1^{\text {st }}$ row). The last column "conditions" provides the nature of the thermal F-S interaction: either moderate/strong interaction $\left(B i_{v}>1\right)$ or weak interaction $\left(B i_{v}<1\right)$. Thus, two zones are identified.

The first zone is characterized by a lower stability limit $\alpha_{f-R}^{\min }$ defined by Eq. (20). The region $\alpha_{f-R}<\alpha_{f-R}^{\min }$ is unstable (see left-hand part of the 3 curves in Figure 2 with $|g|>1$ ). For $\alpha_{f-R}>\alpha_{f-R}^{\min }$, the amplification factor decreases from 1 to a certain limit (given by Eq. (26)) and then increases from this minimum to 1 . This limit is provided by the optimal coefficient (given by Eq. (18)) and corresponds to the absolute minimum of the amplification factor.

The second zone does not exhibit any stability restriction. In other words, all coupled calculations performed with $\alpha_{f-R} \geq 0$ are theoretically stable. The amplification factor has an absolute minimum, namely when $\alpha_{f-R}$ is equal to the optimal coefficient, defined by Eq. (18).

\begin{tabular}{|c|c|c|c|c|c|c|}
\hline$\alpha_{f}$ & $\mathbf{0}$ & & $\alpha_{f-R}^{\min }$ & $\alpha_{f-R}^{(o p t)}$ & $\infty$ & condition \\
\hline$|g|$ & & UNSTA B LE & 1 & $g\left(\alpha_{f-R}^{o p t}\right)$ & 1 & $B i_{v}^{R}>1$ \\
\hline$|g|$ & $B i_{v}^{R}$ & & & $g\left(\alpha_{f-R}^{o p t}\right)$ & 1 & $B i_{v}^{R} \leq 1$ \\
\hline
\end{tabular}

Table 1- Numerical properties of Dirichlet-Robin BC vs. $\alpha_{f}$ 


\section{CODES AND COUPLING LIBRARY}

\subsection{Fluid Code: elsA}

The fluid code, referred to as the elsA software package (ONERA-Airbus-Safran property), is a multi-application CFD simulation platform for applied aerodynamics (internal and external aerodynamics from the low subsonic to the high supersonic flow regime) and multi-physics, which capitalizes on the innovative results of CFD research Erreur! Source du renvoi introuvable.[40][41][43]. The governing equations are the time-dependent Navier-Stokes (NS) equations which express the conservation laws written in the conservation form as

$$
\frac{\partial w_{f}}{\partial t}+\nabla \cdot\left[F\left(w_{f}\right)\right]=0
$$

where $w_{f}$ represents the vector of mass, momentum and energy quantities, $F$ represents the flux including inviscid and viscous terms. The inviscid terms are solved using a second-order upwind spatial discretization. The viscous terms are discretized with a five-point central difference formulation. The time integration is obtained by an implicit method.

\subsection{Solid Code: Z-set}

The solid software package, called Z-set [44], is a comprehensive suite of integrated analysis programs for general purpose structural analysis. Only the thermal solver is employed in the study presented in this paper. Assuming that there are no heat sources, the temperature distribution within the solid body is modelled as a balance of the thermal transport governed by

$$
\rho_{s} C_{s} \partial_{t} T-\nabla \cdot(k . \nabla T)=0
$$

where $T=T(t, x, y, z)$ is the unknown temperature field within the body and $k$ is a thermal conductivity. In the steady-state CHT procedure, we will adopt the steady-state form of (28), i.e. the Laplace's equation $\Delta T=0$.

\subsection{Radiation Code: ASTRE}

ASTRE is a Monte Carlo (MC) radiation transfer code [45]. ASTRE was initially developed to simulate radiative transfers within semi-transparent media and is used mainly to deal with combustion applications [46] or atmospheric reentries [47].

Since ASTRE includes volume-volume, surface-volume and surface-surface radiative exchanges, ASTRE could obviously be used as is, to calculate only the surface-surface (S2S) radiative exchanges in transparent media. However, this would be quite costly in terms of CPU time since the radiative power field would be calculated by default over the entire volume of the computational domain, which is of no use in transparent media. Therefore, ASTRE was recently optimized to deal more efficiently with surface-surface radiative exchanges in transparent media. It would have been very simple to use a view factor calculation capability, but our goal is to develop a methodology with the computation codes available at ONERA in order to be able to apply this methodology to a large variety of complex configurations.

Several MC methods are available in the ASTRE code [48][49]. In this study, the Emission Reciprocity Method (ERM), associated with the Uniform Distribution (UD), was used to calculate the radiative fluxes on the walls. This method is based on the reciprocity principle to calculate 
absorbed fluxes and consequently, it has the advantage of being able to perform local calculations by affecting energy bundles to be emitted only to certain faces of the mesh. This contrasts with the classical MC method that calculates radiative fluxes on all boundaries of the computational domain after the energy bundles emitted from all boundaries are tracked.

In a Monte Carlo computation, a large number of energy bundles is emitted and tracked until they are absorbed. For each energy bundle, two angles are randomly chosen by drawing random numbers. At the end of the simulation, when all the energy bundles have been tracked, the radiative fluxes computed on the walls are fluxes integrated over all directions (see reference [48] for more details).

\subsection{Coupling library}

The coupling between the three aforementioned codes is carried out through the coupling library CWIPI [50], developed by ONERA. CWIPI (Coupling With Interpolation Parallel Interface) is a library that makes it possible to couple an arbitrary number of parallel codes with MPI communications. Coupling is made through an exchange zone that can be discretized in a different way on any coupled code. Linear, surface or volume couplings are available. There is no requirement for the mesh structure, since CWIPI takes into account all types of geometrical elements (polygon, polyhedral).

\section{PARTITIONED LOOSE COUPLING STRATEGIES}

\subsection{Fluid-Radiation algorithm}

The coupling between the fluid code and the radiative code has first been undertaken. All relevant information relating to the physical solvers are transmitted via the coupling library. The coupling scheme is as follows:

- elsA sends to ASTRE the interface temperature

- From this temperature field as BC, ASTRE computes the surface radiative heat flux

- This radiative flux is sent to elsA

- The total heat flux (convective+radiative) is calculated by the fluid code

\subsection{Fluid-Solid-Radiation algorithm}

The sequence of operations in the partitioned procedure is shown in Figure 3 and detailed in Algorithm-1 for the Dirichlet-Robin interface condition. This coupling cycle comprises 7 steps. It can be seen in Figure 3 that it is not exactly a 3-code coupling since the solid solver does not communicate directly with the radiation code.

The process just described is continued until the Navier-Stokes solver converges and wall temperatures and heat fluxes converge, that is until the following tolerance criterion is satisfied

$$
\left\|T_{f}-T_{s}\right\|<\xi
$$




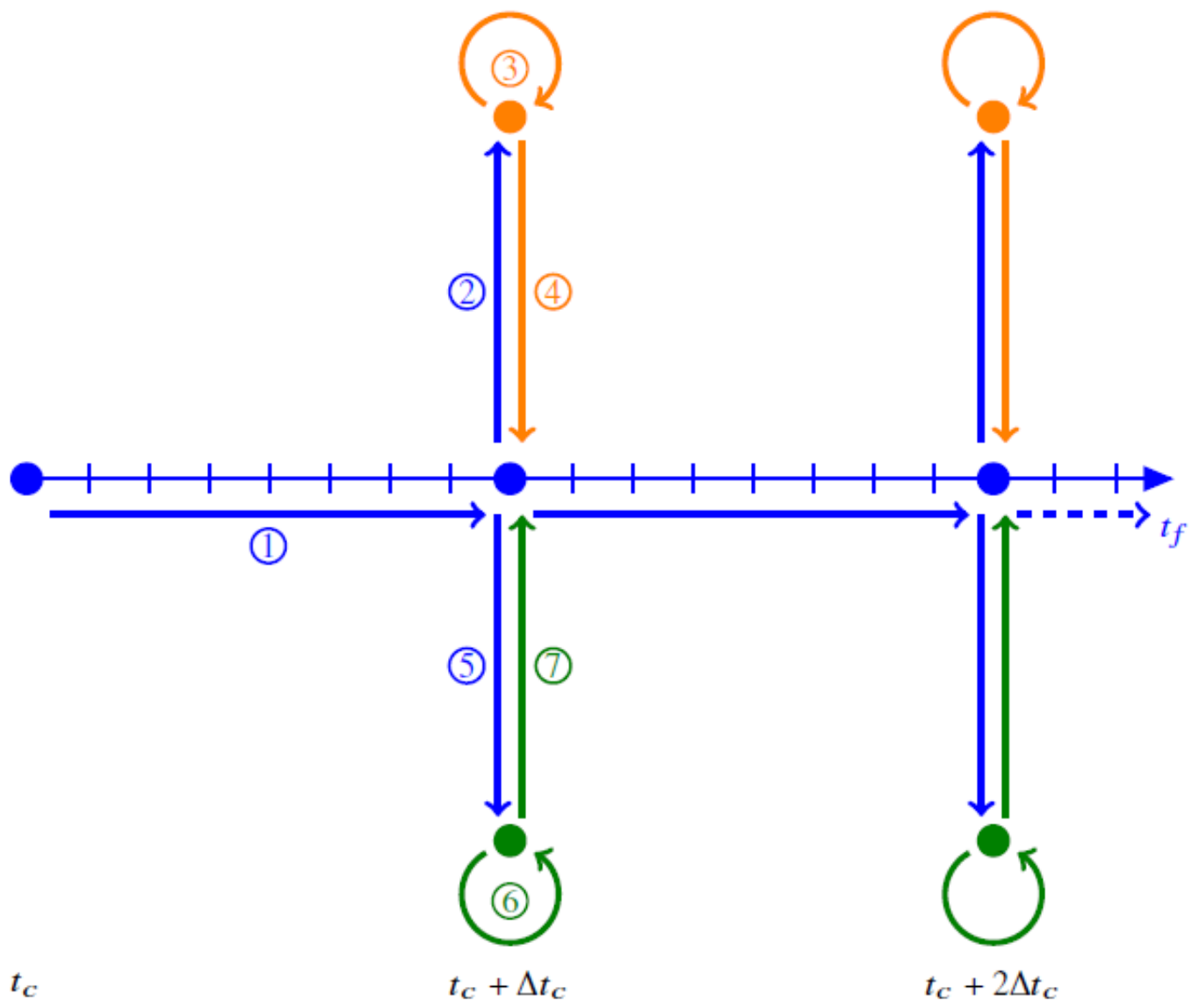

Figure 3 - Algorithm of the Fluid - Solid - Radiation partitioned approach

\footnotetext{
Algorithm 1 - Schematic of the procedure for the fluid-solid-radiation coupling at a given time coupling

(1) The fluid solver elsA computes flow and convective fluxes from time $t_{c}$ to $t_{c}+\Delta t_{c}$

(2) elsA sends the surface fluid temperature distribution to the radiation code ASTRE

(3) ASTRE computes the radiative fluxes using the new wall temperature distribution

(4) ASTRE sends the resulting radiative heat fluxes to elsA

(5) elsA sends to Z-set the total (convective+radiative) heat flux distribution

(6) Z-set solves a steady-state conduction problem

(7) Z-set sends the interface temperature distribution to elsA
} 


\section{AEROTHERMAL TEST CASE WITH RADIATION}

\subsection{Objective of the test case}

In order to illustrate the issues, support the claims made in Section 2 and validate the results, a simple test case with radiating surfaces is considered. Emphasis is put on the destabilizing and stabilizing effects of radiation and the potential of the new optimal coefficient to obtain fast calculations in the presence of radiation. In particular, we would like to check that:

- a stable and fast converging CHT computation can be destabilized only by radiation

- the latter case can be stabilized through the use of the coupling coefficient $\alpha_{f-R}^{\text {opt }}$

- 'optimal' CHT with radiation converges slightly faster than 'optimal' CHT without radiation

\subsection{Choice of the physical and numerical parameters}

The parameters presented in this Section have been purposefully selected to maximize the thermal fluid-structure interaction. To this end, we have performed the procedure listed below in connection with the theoretical concepts just presented. The following three conditions need to be fulfilled:

(1) The fluid-structure coupling is stable and functions ideally without radiation: $\left(\left|g\left(\alpha_{f}^{o p t}\right)\right|<1\right)$ for $\varepsilon=0$.

(2) In a second step, the addition of radiation must destabilize the coupling and the conventional coupling coefficient must become inoperative if it does not take into account radiation $\left(\left|g\left(\alpha_{f}^{\text {opt }}\right)\right|>1\right)$ for a specific value of $\varepsilon>0$. This is theoretically obtained if Condition (25) holds ( $\$ 2.6 .1)$.

(3) In a third step, the new coefficient presented in this study must provide theoretically stable and fast CHT simulations $\left.\left|g\left(\alpha_{f-R}^{o p t}\right)\right|<1\right)$ for the specific value of $\varepsilon$ considered.

As a result, the physical and numerical parameters have been specially chosen to satisfy the above conditions. They are provided in the following Tables 2, 3, 4, 5 and 6.

\begin{tabular}{|c|c|c|c|}
\hline$k_{f}$ & $\Delta y_{f}$ & $K_{f}$ & $\Delta t_{f}$ \\
\hline $6.4710^{-2}$ & $4.010^{-5}$ & 3238 & $2.87510^{-5}$ \\
\hline
\end{tabular}

Table 2 - Fluid parameters

\begin{tabular}{|c|c|c|}
\hline$k_{s}$ & $\Lambda_{s}$ & $K_{s}$ \\
\hline 0.5 & $3.010^{-3}$ & 166.0 \\
\hline
\end{tabular}

Table 3 - Solid parameters

\begin{tabular}{|l|l|l|l|}
\hline$\varepsilon$ & 0. & 0.5 & 0.8 \\
\hline$T_{\text {ref }}$ & \multicolumn{3}{|c|}{1400} \\
\hline$h^{R}$ & 0.0 & 311.0 & 498.0 \\
\hline
\end{tabular}

Table 4 - Radiation parameters 


\begin{tabular}{|c|c|c|c|c|c|c|}
\hline$\Delta t_{c}$ & $D_{f}$ & $\bar{D}_{f}$ & $K_{f} / K_{s}$ & $B i_{v}$ & $\alpha_{f}^{\min }$ & $\alpha_{f}^{\text {opt }}$ \\
\hline $6.010^{-4}$ & 83.0 & 0.856 & 19.43 & 2.78 & 150 & 232 \\
\hline
\end{tabular}

Table 5 - CHT parameters without radiation

\begin{tabular}{|l|l|l|l|}
\hline$h^{R}$ & $B i_{v}$ & $\alpha_{f}^{\min }$ & $\alpha_{f-R}^{\text {opt }}$ \\
\hline 0.0 & 2.78 & 150 & 232 \\
\hline 311.0 & 4.65 & 304 & 540 \\
\hline 498.0 & 5.77 & 395 & 730 \\
\hline
\end{tabular}

Table 6 - CHT parameters with radiation

Let us recall that the above-mentioned parameters have been employed to plot the curves in Figure 2. As a result, these curves may be considered as the theoretical representations of the CHT computations that will be described in this Section.

\subsection{Geometry and boundary conditions}

The turbulent channel configuration is the most frequently used geometry implemented in studies of the near-wall heat transfer and the most accurate approach is conjugate heat transfer. In this study, the geometry is an open 2D channel with a rectangular cross section, where heat conduction inside the heated walls is taken into account. Moreover, the two walls facing one another (coupled interfaces) are radiating. This configuration is displayed in Figure 4.

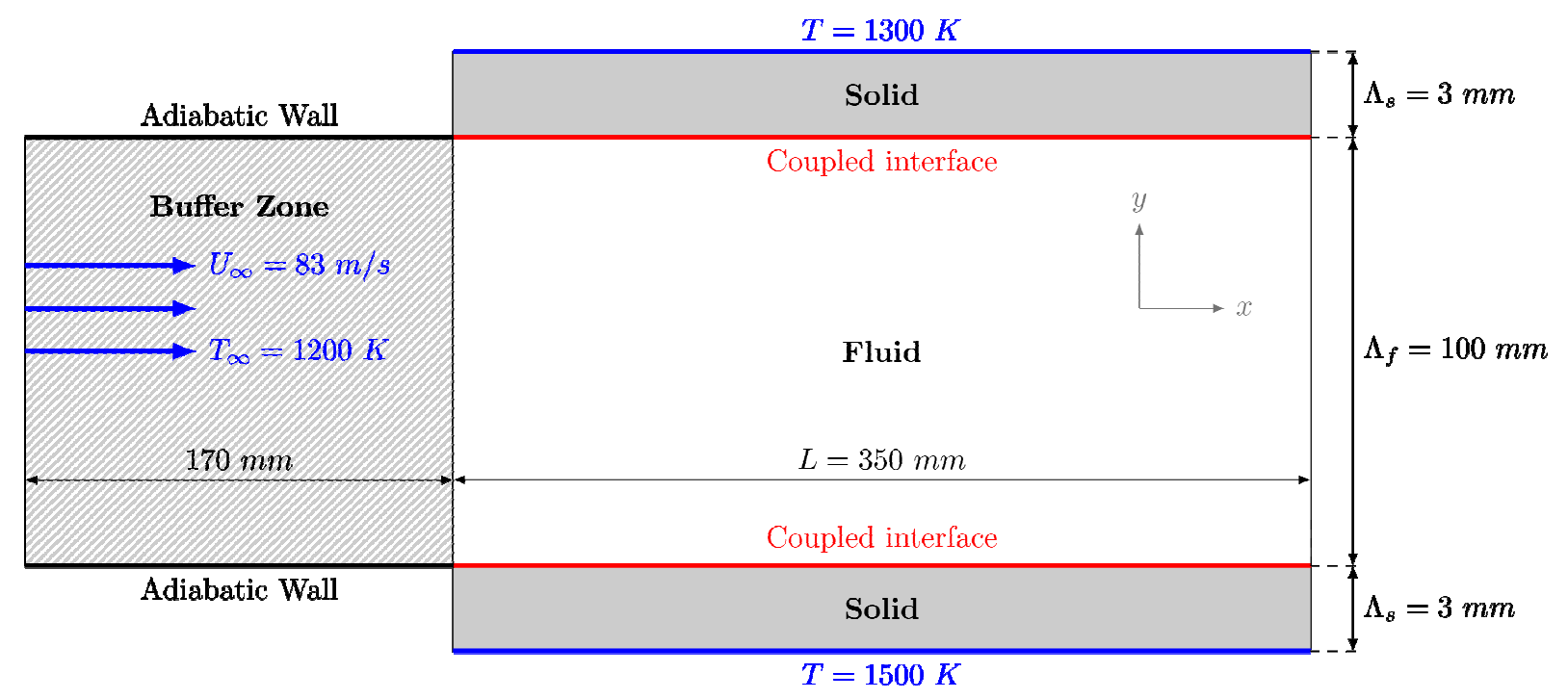

Figure 4 - Sketch of the CHT geometry and boundary conditions

This channel, $350 \mathrm{~mm}$ long x $100 \mathrm{~mm}$ wide, is bounded by two solid plates $3 \mathrm{~mm}$ thick. A temperature of $1300 \mathrm{~K}$ is imposed on the upper surface and $1500 \mathrm{~K}$ is imposed on the lower surface. A buffer zone with upper and lower adiabatic walls is placed upstream to remove the singularity at the leading edges of the flow channel. Turbulent air at a temperature of $1200 \mathrm{~K}$ flows from the inlet (velocity of $83 \mathrm{~m} / \mathrm{s}$ ) to interact and cool the two solid plates (coupled interfaces) before exiting 


\section{A Numerical Predictive Model}

(outlet static pressure: $1,01.10^{5} \mathrm{~Pa}$ ). With these conditions, the Reynolds number, based on the length of the channel, reaches $5.10^{4}$.

Figure 5 shows the mesh of the upper solid, a part of the meshes of the fluid domain and the buffer zone. The fluid mesh has been refined enough in the $y$-direction near the wall $\Delta y_{f}=4.10^{-5} \mathrm{~m}$ to correctly capture the flow boundary layer so that $y^{+} \approx 1$. There is also the same strong refinement at the entrance of the channel flow, $\Delta x_{f}=4 \cdot 10^{-5} \mathrm{~m}$. To avoid interpolation issues in the coupling process, the three meshes (fluid, solid and radiative) are coincident at their shared interface. Each solid domain is composed of rectangular elements, with 19 cells in the $y$-direction according to a geometric progression and 76 surface elements in the interface in the $x$-direction.

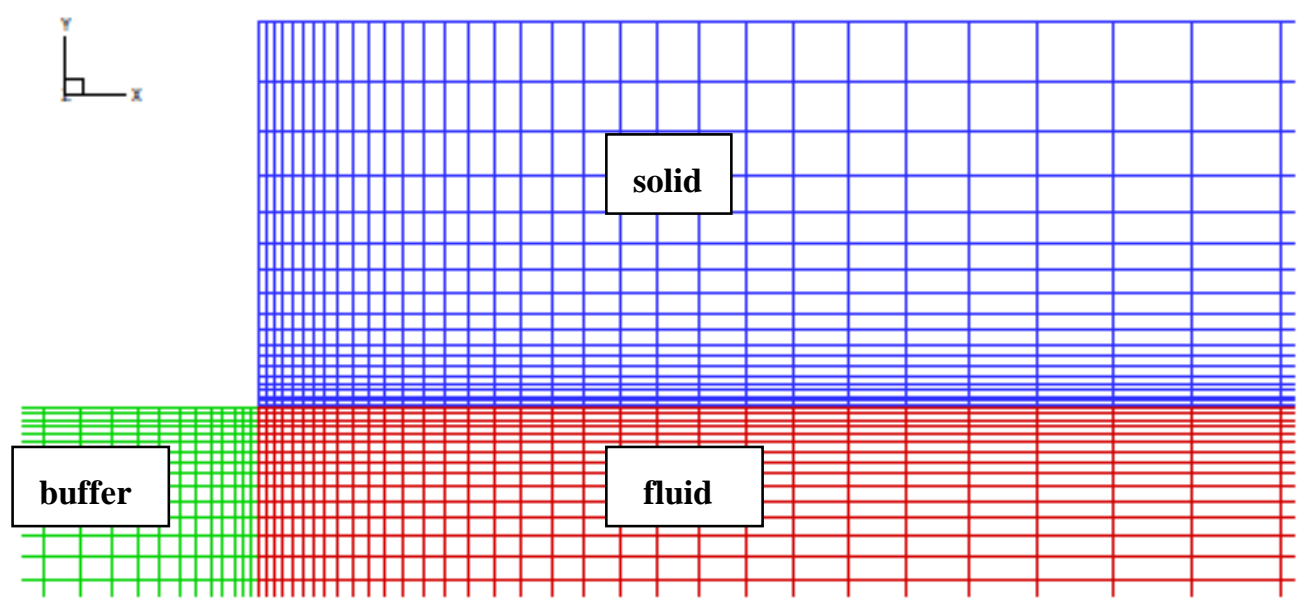

Figure 5 - Parts of the meshes of the fluid, solid and buffer domains

The fluid problem is solved with an Unsteady RANS approach using a Jameson type scheme [51] and the Spalart-Allmaras turbulence model [52]. This unsteady approach is not the most suitable method for quickly obtaining steady-state fluid solutions. However, a constant time step is used throughout the calculation to reproduce the conditions of the model problem. The fluid time step is $\Delta t_{f}=3 \cdot 10^{-5} \mathrm{~s}$. Concerning the calculation of the radiative fluxes, the number of energy bundles, $N_{i}$, emitted by each surface element $i$, was chosen to be 1000 . So, the total number of bundles is $N=152,000$, which appears to be a good compromise between accuracy and calculation time, since a $0.01 \%$ convergence is obtained.

\subsection{Radiative fluxes}

As linear interface conditions are needed in the stability analysis, the radiative flux has been linearized around a reference temperature taken as the average of the initial temperatures of the two walls. However there are two choices. Either the exact formulation is adopted and thus $h^{R}$ (see Sections 2.2.1 and 2.3.1) can be regarded as a local and transient coefficient or a linearization is 


\section{A Numerical Predictive Model}

performed. In the latter case, $h^{R}$ is constant during the whole coupling process. Both formulations are provided in Eq. (7).

It would have been possible to adopt the reference temperature as the temperature of the opposite wall. However, let us recall that the model problem includes only a single fluid-solid interface. Strictly speaking, this means that the opposite wall is not "seen" by this model. In the near future, it could be possible to extend the model to take into account both walls simultaneously.

\subsection{CHT Results}

\subsubsection{Emissivity $\varepsilon=0.5$}

Figure 6 shows the convergence history for two values of the coupling coefficient, by plotting the $L^{\infty}-$ norm of the temperature, i.e. the interface temperature residuals $\Delta T_{f}$ as a function of the coupling iteration. In other words, two identical coupled calculations with radiation (emissivity $\varepsilon=0.5$ ) have been performed. The only difference is the value of the coupling coefficient which, in the first case ignores radiation (optimal coefficient $\alpha_{f}^{(o p t)}$ without radiation given by Eq. (17)) and in the second case takes it into account (optimal coefficient $\alpha_{f-R}^{(o p t)}$ with radiation given by Eq. (18)).

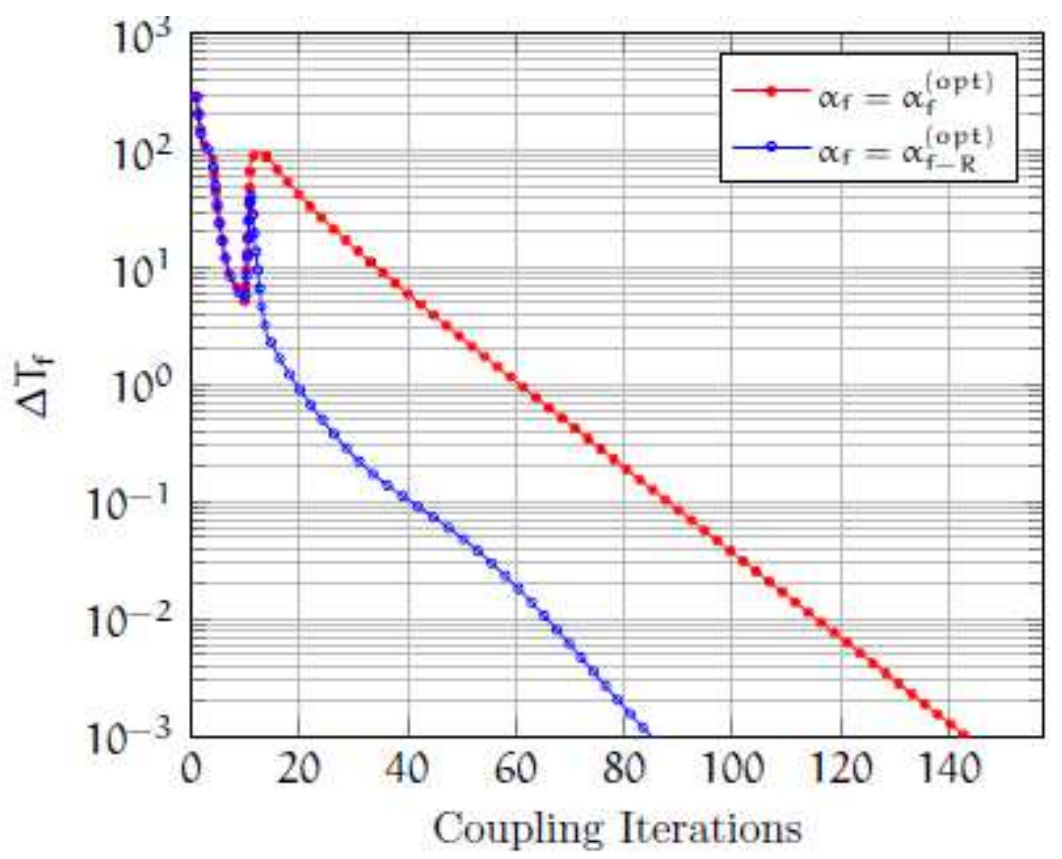

Figure 6 - Convergence history for CHT with radiation $(\varepsilon=0.5)$

The first point is that both calculations converge. During the first 10 coupling iterations the curves are coincident since radiation is introduced in the process at the $11^{\text {th }}$ iteration. The radiation was not set up at the beginning of the coupling process because our objective was to analyze the destabilizing effect of radiation and it was therefore essential to trigger it separately at a predetermined step in the process. At the $11^{\text {th }}$ iteration, a sudden increase in temperature residuals is observed. The peak reached for $\alpha_{f}=\alpha_{f}^{(\text {opt })}$ is the highest and this simulation needs 142 coupling iterations to obtain the 


\section{A Numerical Predictive Model}

convergence $\Delta T_{f}=10^{-3} \mathrm{~K}$. On the contrary, the convergence criterion is satisfied after 84 coupling iterations with $\alpha_{f}=\alpha_{f-R}^{(o p t)}$, i.e. a gain of $69 \%$. Indeed, the calculation with $\alpha_{f}=\alpha_{f-R}^{(o p t)}$ is the fastest CHT process, as predicted by the coupling CHT model presented in Section 2. However, it should be noted that, according to this model, the CHT calculation is supposed to be unstable since $\left|g\left(\alpha_{f}^{o p t}\right)\right|>1$, the absolute minimum of the curve for $\varepsilon=0.5$ in Figure 2. These discrepancies may be due to some stabilizing effects generated by the flow, not included in the theoretical model. It is necessary nevertheless to specify that even if convergence is reached, important oscillations of the interface temperature are observed during the initial phase of the coupling process for this value. However, these oscillations are rapidly damped. On the contrary, for $\alpha_{f}=\alpha_{f-R}^{(o p t)}$, the temperature behavior is oscillation-free. For larger values of $\alpha_{f}$, stable CHT computations are obtained, but they take a little longer. Finally, it is worth mentioning that if the tolerance level is less, the number of iterations is at least cut by half (see for instance in Figure 6, the number of iterations needed to reach $\Delta T_{f}=10^{-1} \mathrm{~K}$ or $\left.\Delta T_{f}=10^{-2} \mathrm{~K}\right)$.

\subsubsection{Emissivity $\varepsilon=0.8$}

Figure 7 illustrates that for a higher value of the radiation $\varepsilon=0.8$, convergence is obtained rapidly when $\alpha_{f}=\alpha_{f-R}^{(\text {opt })}$ is used, with approximately the same number of coupling iterations as before (87 iterations). However, now, the interface temperature residuals increase and as a result, the radiative calculation with $\alpha_{f}=\alpha_{f}^{(o p t)}$ crashes rapidly. One can see from Figure 2 that the amplification factor this time is close to 2 and thus at this stage, nothing can be done to stabilize the coupling process.

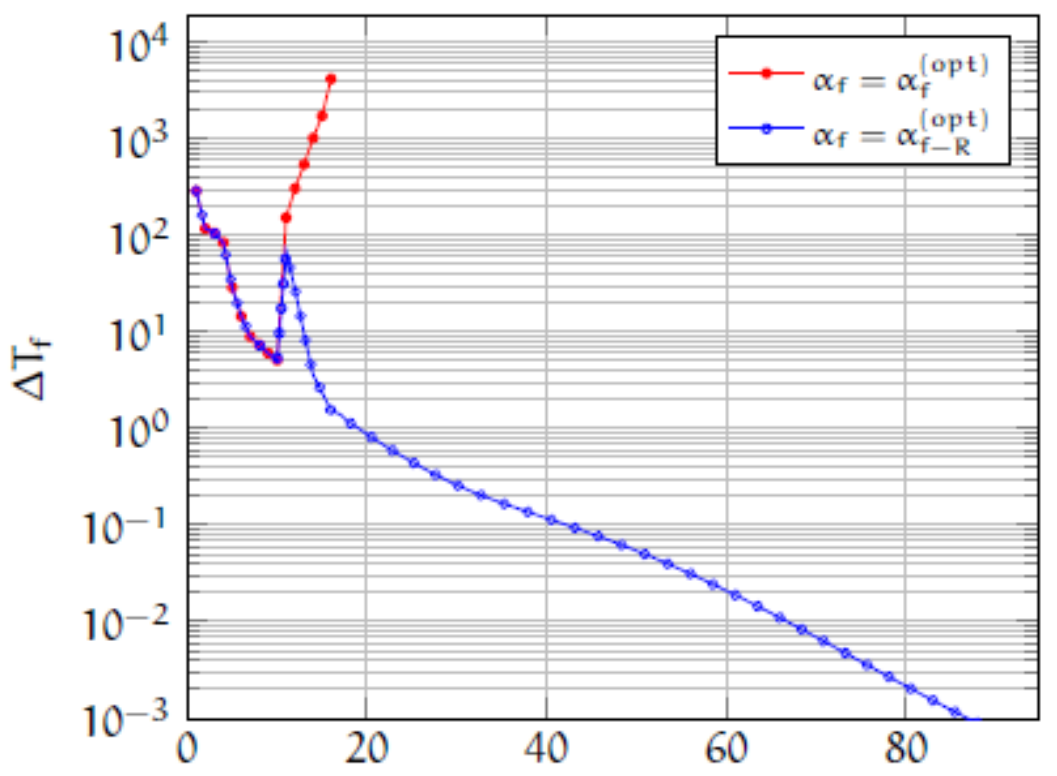

Figure 7 - Convergence history for CHT with radiation $(\varepsilon=0.8)$

To better understand the cause-effect relationships between the coupling model and the coupling results, Table 7 summarizes the key trends of the radiative effect for the three values of emissivity 


\section{A Numerical Predictive Model}

considered and we can see that radiation may play a crucial role. It is thus essential to provide an adequate response to the destabilizing effect of radiation.

\begin{tabular}{|c|c|c|c|c|c|}
\hline$h^{R}$ & $B i_{v}^{R}$ & $\left|g\left(\alpha_{f}^{\text {opt }}\right)\right|$ & $\left|g\left(\alpha_{f-R}^{\text {opt }}\right)\right|$ & $\begin{array}{c}\text { Number of iterations to } \\
\text { converge with } \alpha_{f}^{\text {opt }}\end{array}$ & $\begin{array}{c}\text { Number of iterations to } \\
\text { converge with } \alpha_{f-R}^{\text {opt }}\end{array}$ \\
\hline 0. & 2.78 & 0.58 & 0.58 & 83 & 83 \\
\hline 311. & 4.65 & 1.34 & 0.35 & 142 & 84 \\
\hline 498. & 5.77 & 1.83 & 0.26 & crash & 87 \\
\hline
\end{tabular}

Table 7 - Convergence properties without and with radiation

\subsubsection{Stabilizing effect}

Despite the contradiction with what has just been said, we will now see if the positive effect can be highlighted, as described theoretically in Section 2.6.2. This effect, characterized by a decrease of the temporal amplification factor, should be identified during the convergence history. For that purpose two CHT computations have been compared. The first one without radiation converging in 83 iterations as mentioned in Table 7 and the CHT computation with $\varepsilon=0.8$. Figure 8 depicts the convergence history of these two calculations. During the first 10 iterations, the curves are the same since the radiation has not yet been activated. Then a sudden peak is observed as soon as the radiation is activated. Despite this strong negative effect, the temperature residuals decrease rapidly and the resulting curve always lies below the curve without radiation until the tolerance criterion is satisfied $\left(\Delta T_{f}=10^{-3}\right)$ and hence fits perfectly with the theoretical model. 


\section{A Numerical Predictive Model}

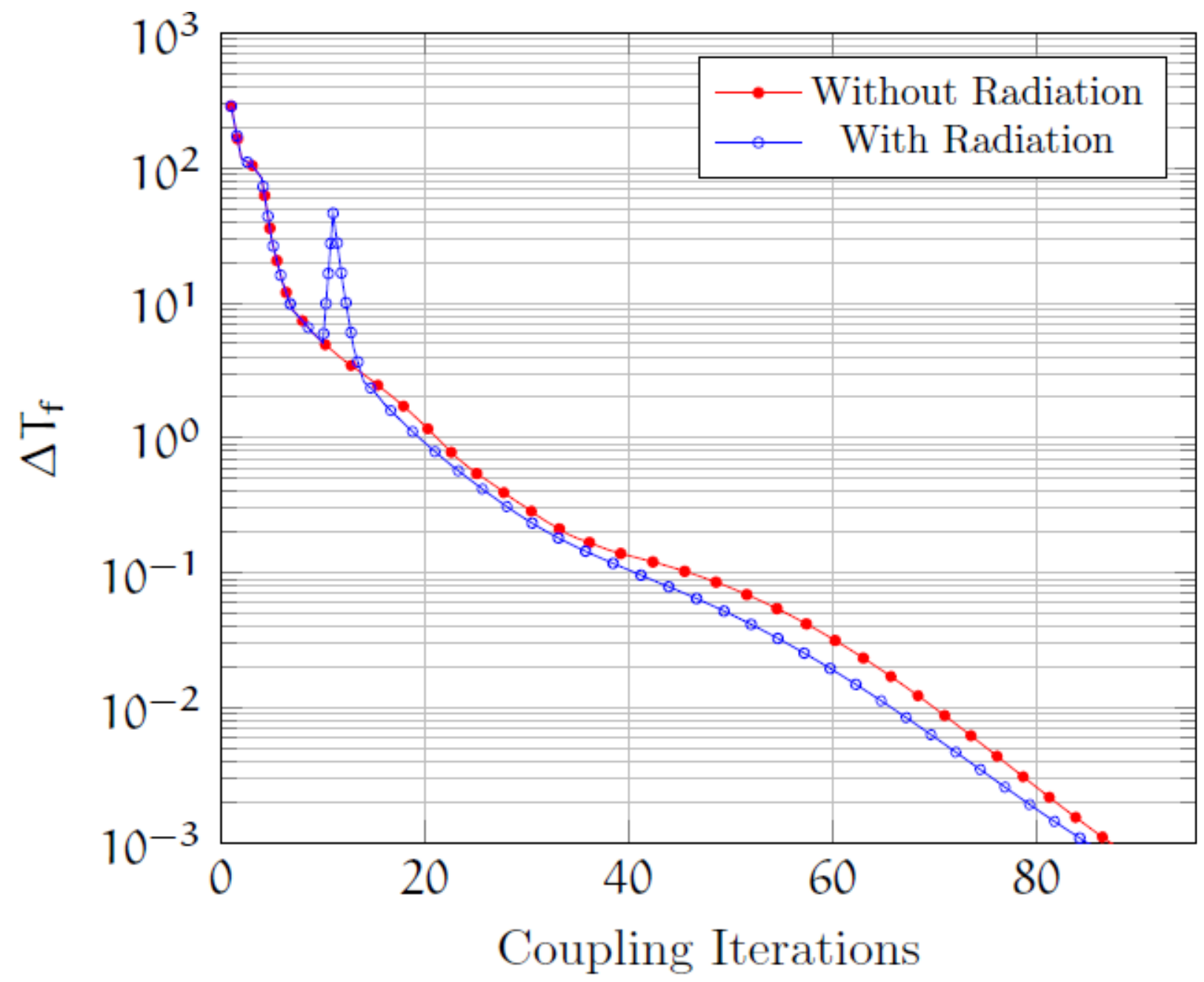

Figure 8 - Comparison of convergence history for CHT with and without radiation

What happens if the tolerance level, adopted until now, becomes more severe? By extending the convergence level, this question is answered in Figure 9. It is interesting to note that the residuals of the CHT computation with radiation end up stagnating around $\Delta T_{f}=10^{-6}$, while the CHT without radiation keeps converging. 


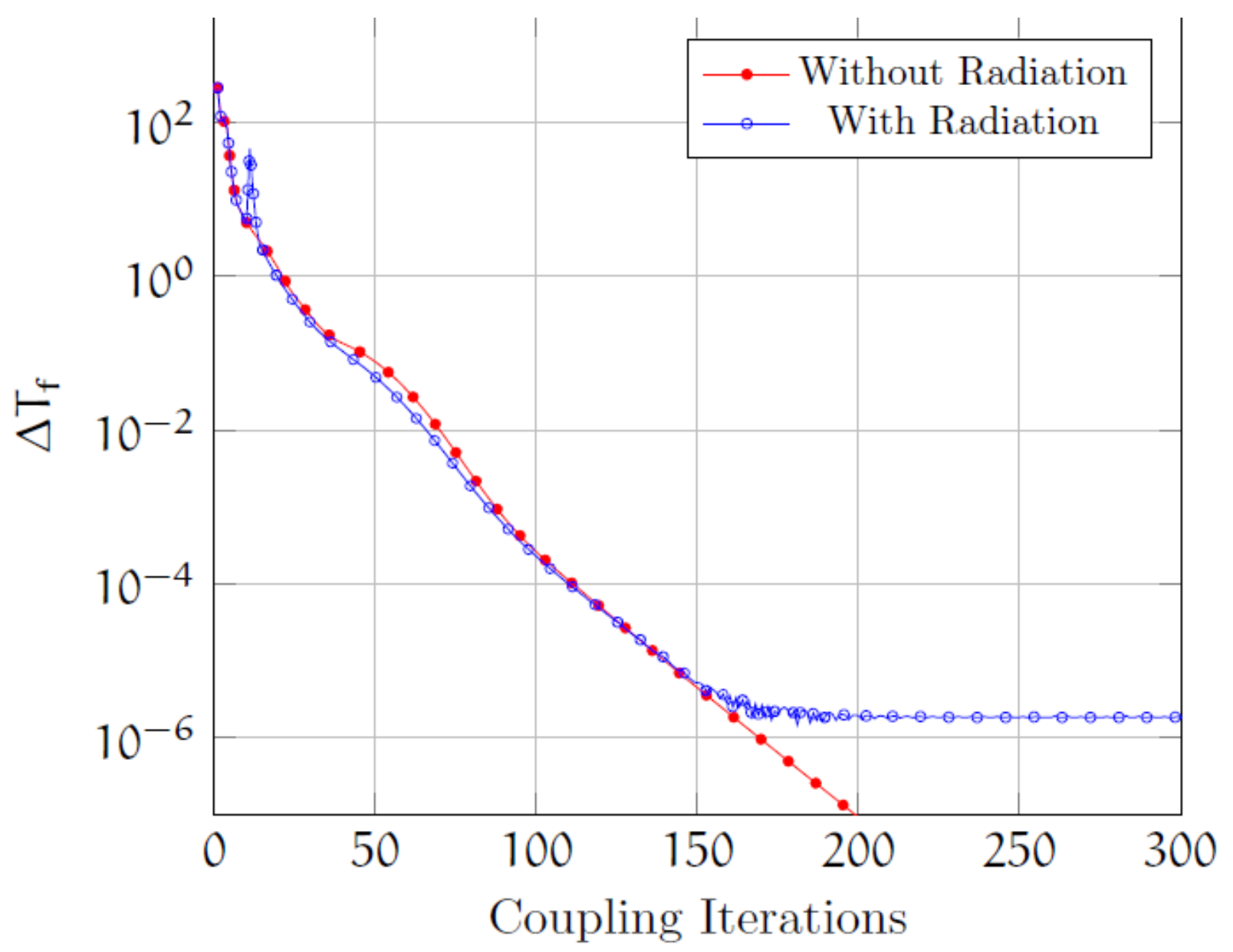

Figure 9 - Comparison of convergence history for CHT with and without radiation

This result does not call into question the mathematical model but, on the contrary, reinforces its validity in so far as the CHT calculation with no radiation can reach very low convergence levels as shown in this Figure and as has been observed in previous studies [29][30]. This is only due to the number, $N$, of energy bundles simulated in the radiation code which automatically limits the convergence level of the entire process.

\subsubsection{Importance of radiation}

The last two figures represent the temperature profiles along the coupling interface (Figure 4) on the upper face (Figure 10) and the lower face (Figure 11). Note that the effect of radiation can be very important and thus not negligible in the thermal design. For example, in this case $(\varepsilon=0.8)$, the lower wall receives a greater amount of heat (an increase of $70 \mathrm{~K}$ or more) when radiation is taken into account. 


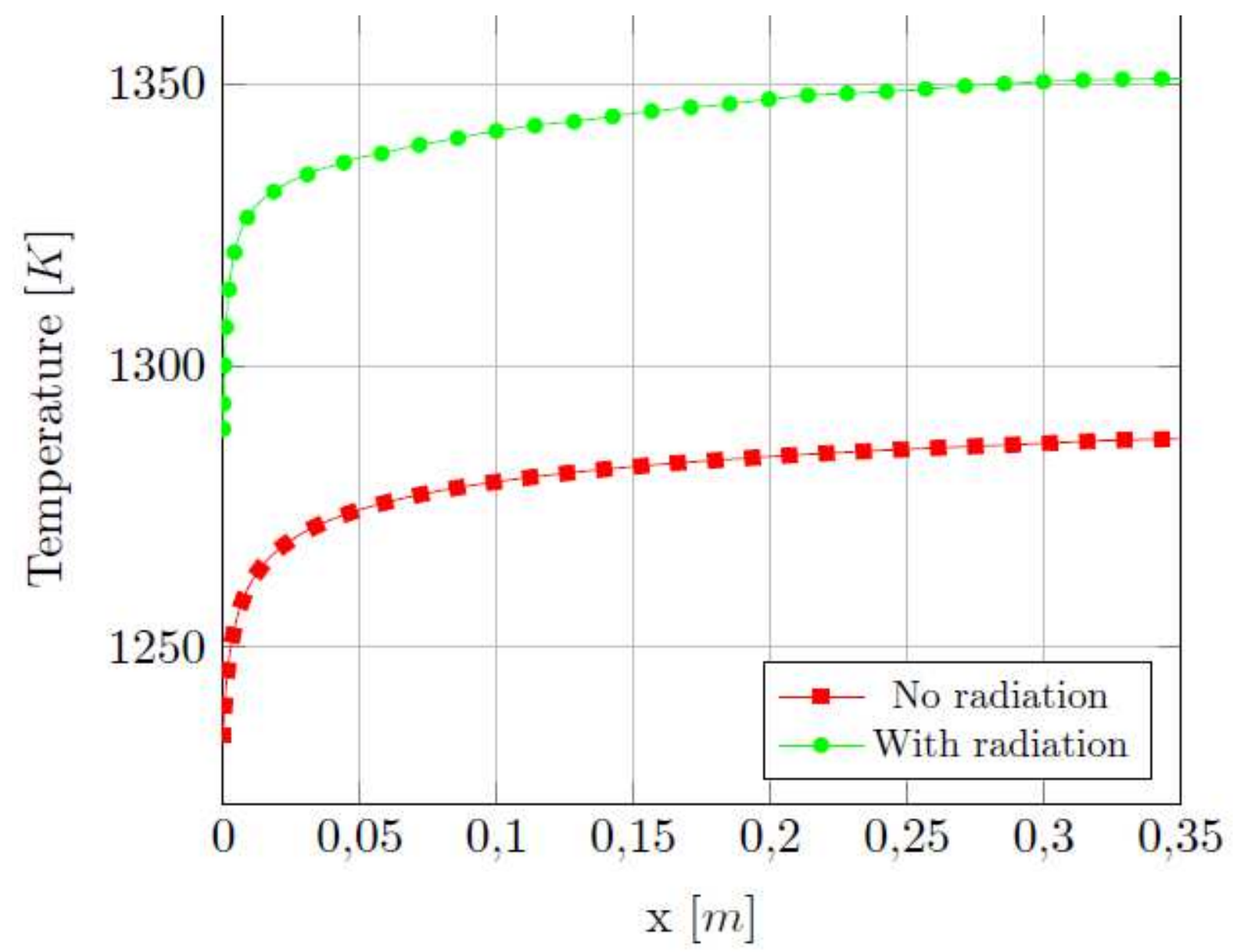

Figure 10 - Temperature profile along the Upper F-S interface at convergence 


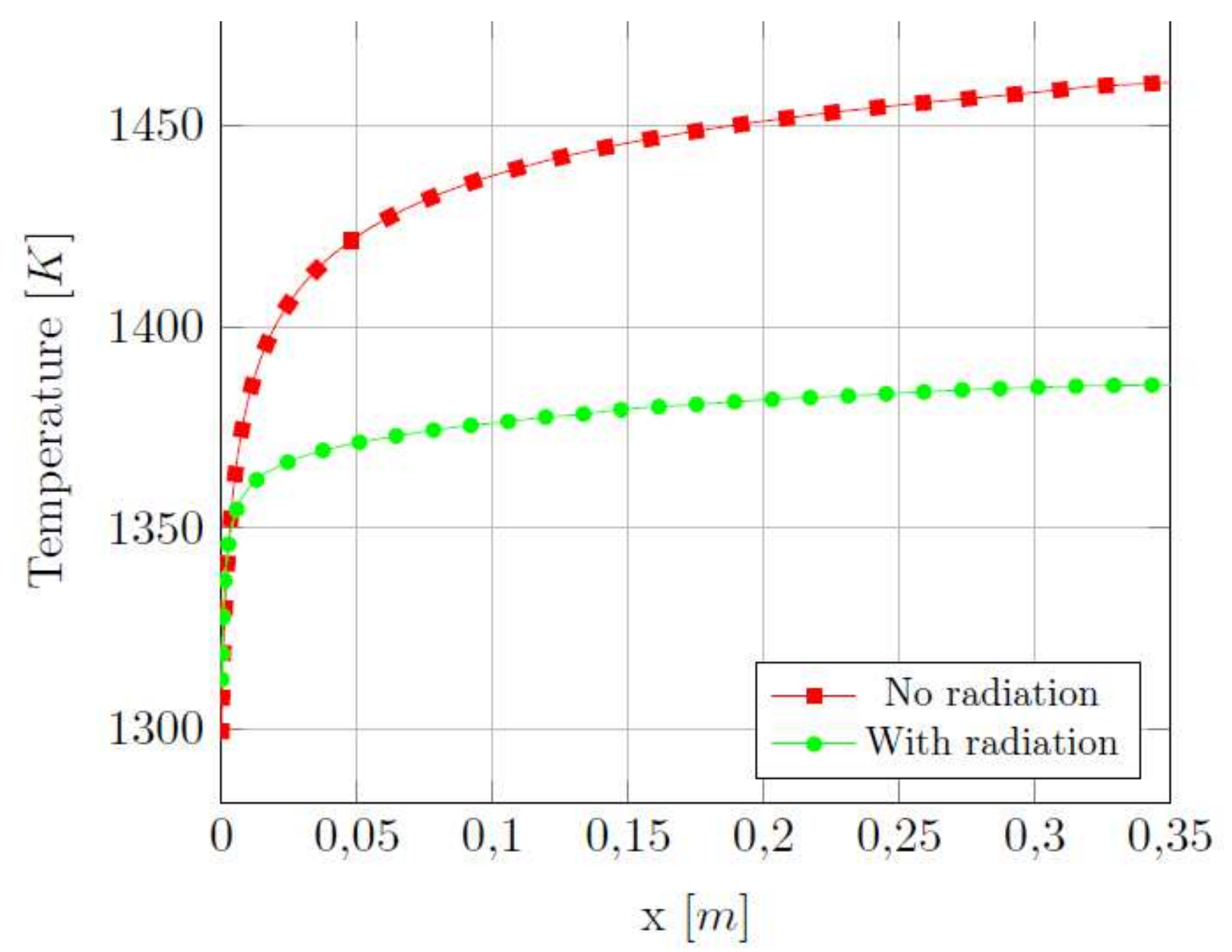

Figure 11 - Temperature profile along the Lower F-S interface at convergence

\subsection{General remarks}

The parameters in this test case have been chosen specifically to provide a significant effect on stability. When this effect has a small impact, the model will obviously still work regardless. However, we now have a fairly good understanding of this behavior and the stability limits are known. Otherwise, when radiation has a moderate or strong destabilizing effect, the situation is more complicated from a stability point of view, but all the relevant settings can now be provided to ensure robust coupled calculations, irrespective of the CHT conditions. The optimal coefficient will adaptively adjust to the local conditions. In any case, whether the radiation impact is big or small, optimization in terms of convergence and CPU time will be automated to a certain degree in a fairly straightforward manner. 


\section{CONCLUSION}

A predictive coupling model for steady conjugate heat transfer problems with radiation was proposed in this paper. It was derived from a stability analysis based on the Godunov-Ryabenkii normal mode theory. The stability bounds, the optimal coefficients and the main numerical characteristics of the interface procedures for aerothermal interaction with radiation were highlighted and expressed. The numerical treatment relies on adaptive and local coefficients. Consequently, arbitrary relaxation parameters are no longer required in the coupling procedure and oscillation-free coupled solutions can be obtained. The model presented in this article is part of a wider strategy to ensure optimal treatments for CHT problems. It was shown that radiation in a transparent medium with gray walls can be taken into account in a simple and effective manner. In the near future, consideration should be given to extending this model to semi-transparent media.

In the CHT test case, emphasis was put on the destabilizing nature of radiation and the main results derived from the mathematical model were confirmed. All the key trends of radiation effects identified theoretically were recovered and the potential of the new optimal coefficient to obtain fast calculations in the presence of radiation was confirmed. It was also checked that 'optimal' CHT radiation converges slightly faster, which might seem surprising. However, the non-linear nature of the thermal radiation should not be forgotten and thus the reliability of the use of a linearized radiative coefficient could be questioned or invalidated under some circumstances. Nonetheless, the significant patterns and trends illustrated in this paper are particularly valuable in a wide range of engineering applications where combined heat transfer of radiation, convection/conduction is encountered. 
A Numerical Predictive Model

\section{Acknowledgements}

The authors are grateful to the "Direction Scientifique Générale" of ONERA for their ongoing support. A huge thank you is addressed to Andrew Mayne for proof-reading and correcting with great care this article. 


\section{APPENDIX A}

\section{Single interface equation for the Dirichlet-Robin boundary condition with radiation}

The goal of this Appendix is to determine a single interface equation for the Dirichlet-Robin boundary condition with radiation. The temporal interval $[n, n+1]$ in a sequential algorithm is considered at the fluid-solid interface. It must be recalled here that $(\hat{X})$ denotes the sought value $X$.

At time $t^{n}$, on the fluid side, a Robin interface condition (see Eq. 1) has the following form :

$$
\hat{q}_{f}^{n+1}+\alpha_{s}^{n} \hat{T}_{f}^{n+1}+\left(\hat{\Phi}_{f}^{R}\right)^{n+1}=q_{s}^{n}+\alpha_{s}^{n} T_{s}^{n}
$$

After completing the previous step, the fluid quantities are transferred to the solid and implemented on the right-hand side. A Robin equation is employed on the solid side of the same interface at time $t^{n+1}$ (see Eq. 2)

$$
\hat{q}_{s}^{n+1}-\alpha_{f}^{n+1} \hat{T}_{s}^{n+1}=q_{f}^{n+1}-\alpha_{f}^{n+1} T_{f}^{n+1}+\left(\Phi_{s}^{R}\right)^{n+1}
$$

The radiative fluxes are linearized (see Eq. 9) and thus the interface conditions become

$$
\left\{\begin{array}{l}
\hat{q}_{f}^{n+1}+\left(\alpha_{s}^{n}+h_{f}^{R}\right) \hat{T}_{f}^{n+1}=q_{s}^{n}+\alpha_{s}^{n} T_{s}^{n}+h_{f}^{R} T_{r e f} \\
\hat{q}_{s}^{n+1}-\alpha_{f}^{n+1} \hat{T}_{s}^{n+1}=q_{f}^{n+1}-\alpha_{f}^{n+1} T_{f}^{n+1}+h_{s}^{R}\left(T_{f}^{n+1}-T_{\text {ref }}\right)
\end{array}\right.
$$

with $\Phi_{s}^{R}=h_{s}^{R}\left(T_{f}-T_{r e f}\right)$ and $\Phi_{f}^{R}=h_{f}^{R}\left(T_{f}-T_{r e f}\right)$.

The system (A-3) represents the general formulation of the Robin-Robin interface conditions. From now on, we will consider only a Dirichlet boundary condition in the fluid domain. This condition is directly obtained by imposing $\alpha_{s}=\infty$. Then, expressing the heat fluxes in terms of the temperature, (A-3) becomes

$$
\left\{\begin{array}{l}
\hat{T}_{f}^{n+1}=T_{s}^{n} \\
{\left[-K_{s}^{n} \frac{\partial}{\partial v}-\alpha_{f}^{n+1}\right] \hat{T}_{s}^{n+1}=-K_{f}^{n+1} \frac{\partial T_{f}^{n+1}}{\partial v}-\left(\alpha_{f}^{n+1}-h_{s}^{R}\right) T_{f}^{n+1}-h_{s}^{R} T_{r e f}}
\end{array}\right.
$$

Where $v$ is the unit normal vector at the fluid-solid interface, i.e. pointing to the inward normal of the fluid (see Figure 1). For easier reading, the superscripts $n$ and $n+1$ will now be removed in the coefficients $K_{f}, K_{s}$, and $\alpha_{f}$.

At this stage, it is worth remembering that the temperatures $T_{s}$ and $T_{f}$ are located on either side of the same interface $y=0$. In other words, $T_{s}=T_{0-}$ and $T_{f}=T_{0+}$. As the derivatives $\frac{\partial}{\partial v}$ at the interface are approximated by one-sided differences, (A-4) becomes

$$
\left\{\begin{array}{l}
\hat{T}_{0+}^{n+1}=T_{0-}^{n} \\
\left(K_{s}+\alpha_{f}\right) \hat{T}_{0-}^{n+1}-\frac{\lambda_{s}}{\Delta y_{s}} \hat{T}_{-1}^{n+1}=\left(\alpha_{f}-K_{f}-h_{s}^{R}\right) T_{0+}^{n+1}+K_{f} T_{1}^{n+1}+h_{s}^{R} T_{r e f}
\end{array}\right.
$$

Since a steady-state is considered on the solid side, $\frac{T_{0-}-T_{-1}}{\Delta y_{s}}=\frac{T_{0-}-T_{-J}}{\Lambda_{s}}$. Thus (A-5) can be rewritten

$$
\left\{\begin{array}{l}
\hat{T}_{0+}^{n+1}=T_{0-}^{n} \\
\left(\beta K_{s}+\alpha_{f}\right) \hat{T}_{0-}^{n+1}=\left(\alpha_{f}-K_{f}-h_{s}^{R}\right) T_{0+}^{n+1}+K_{f} T_{1}^{n+1}+\beta K_{s} T_{-J}+h_{s}^{R} T_{r e f}
\end{array}\right.
$$

This system becomes 


$$
\left\{\begin{array}{l}
\hat{T}_{0+}^{n+1}=T_{0-}^{n} \\
\left(\beta K_{s}+\alpha_{f}\right) \hat{T}_{0-}^{n+1}=\left(\alpha_{f}-K_{f}-h_{s}^{R}\right) T_{0+}^{n+1}+K_{f} T_{1}^{n+1}+G_{e x t}
\end{array}\right.
$$

Where $G_{e x t}$ is a constant term defined by $G_{e x t}=\beta K_{s} T_{-J}+h_{s}^{R} T_{r e f}$.

Let us remind here, that the coefficient $\beta$ is a dimensionless number that can be thought of as a simplified representation of the outer boundary condition $-J$ of the solid domain. If a temperature is imposed at this limit, then $\beta=1$. See [26] for more details.

Substituting the definition of $T_{0+}^{n+1}$ into the second equation of (A-7), we find

$$
\left(\beta K_{s}+\alpha_{f}\right) \hat{T}_{0-}^{n+1}=+K_{f} T_{1}^{n+1}+\left(\alpha_{f}-K_{f}-h_{s}^{R}\right) T_{0-}^{n}+G_{e x t}
$$

We therefore arrive at

$$
T_{0-}^{n+1}=\frac{K_{f}}{K} T_{1}^{n+1}+\frac{\left(\alpha_{f}-K_{f}-h_{s}^{R}\right)}{K} T_{0-}^{n}+\frac{G_{e x t}}{K}
$$

At this point, it must be emphasized that a constant plays no role in the normal mode stability analysis and thus the term $G_{e x t}$ can be removed. Finally, the following single interface equation is obtained

$$
\begin{aligned}
T_{0-}^{n+1} & =A T_{1}^{n+1}+B T_{0-}^{n} \\
\text { With } \quad A=\frac{K_{f}}{K} \quad, \quad B & =\frac{\left(\alpha_{f}-K_{f}-h_{s}^{R}\right)}{K} \quad, \quad K=\beta K_{s}+\alpha_{f}
\end{aligned}
$$




\section{APPENDIX B}

\section{Stability Analysis}

\section{B.1 Link between the fluid and solid temporal amplification factor}

The stability analysis is based on the Godunov \& Ryabenkii theory. We seek normal mode solutions to the equation (A-10) of the form

$$
T_{j}^{n}= \begin{cases}z_{f}^{n-1} \kappa_{f}^{j}, & j>0 \\ z_{s}^{n} \kappa_{s}^{j}, & j \leq 0\end{cases}
$$

Where $\mathrm{z}$ is the "temporal" amplification factor and $\kappa$ is the "spatial" amplification factor.

Substitution of the normal mode solution (B-1) into the continuity of temperature at the fluid-solid interface $\left(T_{0+}^{n+1}=T_{0-}^{n}\right)$ leads to

$$
z_{f}^{n}=z_{s}^{n} \Rightarrow\left|z_{f}\right|=\left|z_{s}\right|=|z|
$$

\section{B.2. Characteristic equation in the steady solid domain}

Introducing the second equation of (B-1) into the Laplace equation solved in the solid domain yields

$$
\kappa_{s}^{2}-2 \kappa_{s}+1=0 \quad j \leq 0
$$

Thus the trivial solution $\kappa_{s}=1$ is obtained, which means that there is no spatial amplification in the solid domain.

\section{B.3. Characteristic equation in the transient fluid domain}

In the interval $[n, n+1]$, the fluid solution is advanced from $t^{n}$ to $t^{n+1}$ and a new temperature field is calculated on the basis of a forward implicit scheme. In the first grid cell adjacent to the interface, the following discrete equation is solved

$$
T_{j+1}^{n+1}-T_{j+1}^{n}=D_{f}\left(T_{j+1}^{n+1}-2 T_{j}^{n+1}+T_{j}^{n+1}\right)
$$

Substitution of the normal mode solution (B-1) into this equation leads to

$$
\kappa_{f}^{2}-\left(2+\frac{z-1}{D_{f} z}\right) \kappa_{f}+1=0
$$

There is only one acceptable solution to this equation, given by the choice of the root with the minus sign

$$
\kappa_{f}\left(D_{f}, z\right)=\frac{1}{2}\left(2+\frac{z-1}{D_{f} z}-\sqrt{\left(2+\frac{z-1}{D_{f} z}\right)^{2}-4}\right)
$$

From (B-6), we can see that the values of the complex function, $\kappa_{f}$, at the specific points $z=+1$ and $z=-1$ are real and equal to

$$
\left\{\begin{array}{l}
\kappa_{f}\left(D_{f}, z=+1\right)=1 \\
\kappa_{f}\left(D_{f}, z=-1\right)=\bar{D}_{f}
\end{array}\right.
$$

The definition of the normalized Fourier number $\bar{D}_{f}$ is given by (15). 
B.4. Temporal amplification factor of the coupled problem

Now, inserting the normal mode solution (B-1) into the single interface condition given in Equation (A-10), we readily obtain the temporal amplification factor for the coupled model problem

$$
z=g\left(z, \alpha_{f}\right)=A \kappa_{f}\left(D_{f}, z\right)+B
$$

With $\kappa_{f}$ given by (B-6). The coefficients A and B are defined by (A-11).

\section{B.5. Application to the Dirichlet-Robin interface condition}

\section{B.5.1. Temporal Amplification factor}

From (B-8) and (A-11), it is straightforward to obtain the temporal amplification factor

$$
g\left(z, \alpha_{f}\right)=\frac{K_{f}}{\beta K_{s}+\alpha_{f}} \kappa_{f}\left(D_{f}, z\right)+\frac{\left(\alpha_{f}-K_{f}-h_{s}^{R}\right)}{\beta K_{s}+\alpha_{f}}
$$

The expression given by (13) is recovered

\section{B.5.2. Spatial amplification factor}

From (A-10) we obtain

$$
\frac{T_{0-}^{n+1}}{T_{0-}^{n}}=A \frac{T_{1}^{n+1}}{T_{0-}^{n}}+B
$$

The left-hand side of equation (B-10) represents the definition of the temporal amplification factor $z$. As a result, from (B-8), another simple expression of the spatial amplification factor in the fluid domain may readily be expressed

$$
\kappa_{f}=\frac{T_{1}^{n+1}}{T_{0-}^{n}}=\frac{T_{1}^{n+1}}{T_{0+}^{n+1}}
$$

\section{B.5.3. Optimal coefficient}

The amplification factor is composed of two half-lines with a singular point at the intersection of these two lines defined by

$$
\left|g\left(z=1, \alpha_{f}\right)\right|=\left|g\left(z=-1, \alpha_{f}\right)\right|
$$

Using the definition given by (B-8) in its simple form, (B-12) becomes

$$
\left|A \kappa_{f}\left(D_{f}=1, z\right)+B\right|=\left|A \kappa_{f}\left(D_{f}=-1, z\right)+B\right|
$$

Substituting the definition of $\kappa_{f}$ given by (B-7), (B-13) becomes

$$
|A+B|=\left|A \bar{D}_{f}+B\right|
$$

Thus, a remarkable condition for a transition to occur is obtained for

$$
\left(1+\bar{D}_{f}\right) \cdot K_{f}+2 \cdot\left(\alpha_{f}-K_{f}-h_{s}^{R}\right)=0
$$

The unique coefficient satisfying (B-15), is called an optimal coefficient since the temporal amplification factor attains an absolute minimum on the unit circle. From (B-15) we obtain 


$$
\alpha_{f}=\alpha_{f-R}^{o p t}=\frac{K_{f}}{2}\left(1-\bar{D}_{f}\right)+h_{s}^{R}
$$

The expression given by (18) is recovered.

\section{B.5.4. Stability limit}

The stability bound is obtained for $\left|g\left(z, \alpha_{f}, \alpha_{s}\right)\right|=1$. Thus from (B-8)

$$
\left|A \kappa_{f}\left(D_{f}, z\right)+B\right|=1
$$

This relationship may occur only in the unstable zone and thus $z=-1$ and $\kappa_{f}=\bar{D}_{f}$. As a result (B-17) becomes

$$
A \bar{D}_{f}+B=-1
$$

And consequently

$$
K_{f} \bar{D}_{f}-\left(K_{f}-\alpha_{f}+h_{s}^{R}\right)=-\left(\beta K_{s}+\alpha_{f}\right)
$$

Finally, the stability limit is

$$
\alpha_{f-R}^{\min }=\frac{K_{f}}{2}\left(1-\bar{D}_{f}\right)+\frac{h_{s}^{R}}{2}-\frac{\beta K_{s}}{2}
$$

The expression given by (19) is recovered. 


\section{REFERENCES}

[1] T.L. Perelman, On conjugated problems of heat transfer, Int. J. Heat Mass Transfer 3 (1961) 293303. https://doi.org/10.1016/0017-9310(61)90044-8.

[2] A.V. Luikov, T.L. Perelman, R.S. Levitin, L.B. Gdalevitch, Heat transfer from a plate in a compressible gas flow, Int. J. Heat Mass Transfer 13 (1971) 1261-1270. https://doi.org/10.1016/0017-9310(70)90067-0

[3] M.B. Giles, Stability Analysis of Numerical Interface Conditions in Fluid-Structure Thermal Analysis, International Journal for Numerical Methods in Fluids, 25, pp. 421-436, 1997.

[4] B. Roe, R. Jaiman, A. Haselbacher, and P.H. Geubelle, Combined interface boundary method for coupled thermal simulations, Int. J. Numer. Meth. Fluids 57, pp. 329-354, 2008.

[5] W.D. Henshaw, and K.K. Chand, A composite grid solver for conjugate heat transfer in fluidstructure systems, Journal of Computational Physics 228, pp 3708-3741, 2009.

[6] V. Kazemi-Kamyab, A.H. van Zuijlen, and H. Bijl, Accuracy and stability analysis of a secondorder time-accurate loosely coupled partitioned algorithm for transient conjugate heat transfer problems, Int. J. Numer. Meth. Fluids, DOI: 10.1002/fld.3842, 2013.

[7] O. Joshi and P. Leyland, Stability Analysis of a Partitioned Fluid-Structure Thermal Coupling Algorithm, Journal of Thermophysics and Heat Transfer, Vol. 28, N 1 , January-March 2014.

[8] J. Lindström, and J. Nordström, A stable and high-order conjugate heat transfer problem, Journal of Computational Physics 229, pp 5440-5456, 2010.

[9] F.-X. Roux and J.-D. Garaud, Domain Decomposition Methods Methodology with Robin Interface Matching Conditions for Solving Strongly Coupled Fluid-Structure Problems, International Journal for Multiscale Computational Engineering, 7, Issue 1, pp. 9-16, 2009.

[10] T. Verstraete and S. Scholl, Stability analysis of partitioned methods for predicting conjugate heat transfer, International Journal of Heat and Mass Transfer, 2016, 101, 852-869.

[11] L. He and M.L.G. Oldfield, Unsteady conjugate heat transfer modeling, Journal of Turbomachinery, Vol. 133, N³, 2011.

[12] F. Duchaine, A. Corpron, L. Pons, V. Moureau, F. Nicoud, T. Poinsot, Development and Assessment of a Coupled Strategy for Conjugate Heat Transfer with Large Eddy Simulation: Application to a Cooled Turbine Blade, Int. J. Heat Fluid Flow, 30(6), pp.1129-1141, 2009.

[13] S. Jaure, F. Duchaine, G. Staffelbach and L.Y.M Gicquel, Massively parallel Conjugate Heat Transfer Methods relying on Large Eddy Simulation applied to an Aeronautical Combustor, Computational Science \& Discovery 6 - 015008, 2013.

[14] L. He and M. Fadl, Multi-scale time integration for transient conjugate heat transfer, International Journal for Numerical Methods in Fluids, Vol. 83, 12, pp 887-904, 2016.

[15] C. Koren, R. Vicquelin, O. Gicquel, Self-adaptive coupling frequency for unsteady coupled conjugate heat transfer simulations, International Journal of Thermal Sciences, 118, pp.340- 354, 2017.

[16] R. Goulard and M. Goulard, Energy transfer in Couette flow of a radiant and chemically reacting gas, Proc. Heat Transf. Fluid Mech. Inst. Stanford University Press, Stanford, 1959.

[17] V. N. Adrianov and S.N. Shorin, Radiant heat transfer in a flowing radiating medium, AIAA Journal, Russian supplement, Vol.1, N7, July 1963, https://doi.org/10.2514/3.1914.

[18] T. H. Einstein, Radiant heat transfer to absorbing gases enclosed in a circular pipe with conduction, gas flow, and internal heat generation, NASA TR R-156, 1963.

[19] S. Desoto, Coupled radiation, conduction and convection in entrance region flow. Int. J. Heat Mass Transf. Vol. 11. pp. 39-53. Pergamon Press 1968. 
[20] J. Amaya, 2010. Unsteady coupled convection, conduction and radiation simulations on parallel architectures for combustion applications. PhD thesis, CERFACS.

[21] J. Amaya, O. Cabrit, D. Poitou, B. Cuenot, M. E Hafi, Unsteady coupling of Navier-Stokes and radiative heat transfer solvers applied to an anisothermal multicomponent turbulent channel flow. J. Quant. Spec. Rad. Transf. 111(2), 295-301. 2010.

[22] F. Duchaine, , A. Corpron, L. Pons, V. Moureau, F. Nicoud, and T. Poinsot, Development and assessment of a coupled strategy for conjugate heat transfer with Large Eddy Simulation. Application to a cooled turbine blade. International Journal of Heat and Mass Transfer, Volume 30, Issue 6, pp 1129-1141, 2009.

[23] F. Duchaine, S. Jauré, D. Poitou, E. Quémerais, G. Staffelbach, T. Morel and L. Gicquel, Analysis of high performance conjugate heat transfer with the OpenPALM coupler, Computational Science \& Discovery 8(1), July 2015.

[24] C. Koren, R. Vicquelin, O. Gicquel, Multiphysics Simulation Combining Large-Eddy Simulation, Wall Heat Conduction and Radiative Energy Transfer to Predict Wall Temperature Induced by a Confined Premixed Swirling Flame, Applied Scientific Research, 101(1), 77-102, 2018.

[25] E. Mercier, L. Tessé, N. Savary, 3D full predictive thermal chain for gas turbine combustor metal temperature, Proceedings of the 25th International Congress of the Aeronautical Sciences (ICAS 2006), Hamburg, Germany, 3-8 September 2006.

[26] M.-P. Errera and S. Chemin, Optimal solutions of numerical interface conditions in fluidstructure thermal analysis, Journal of Computational Physics, 245, pp 431-455, 2013.

[27] M.-P. Errera, F. Duchaine, Comparative study of coupling coefficients in Dirichlet-Robin procedure for fluid-structure aerothermal simulations, J. Comput. Phys. 312 (2016) 218-234. doi:10.1016/j.jcp.2016.02.022

[28] B. Cuenot, Robin procedure for fluid-structure aerothermal simulations, Journal of Computational Physics, 312, 218-234, 2016.

[29] R. Moretti, M.-P. Errera, V. Couaillier, F. Feyel, Stability, convergence and optimization of interface treatments in weak and strong thermal fluid-structure interaction, International Journal of Thermal Sciences, 126, pp 23-37, 2018.

[30] M-P. Errera, M. Lazareff, J-D. Garaud, T. Soubrié, C. Douta, T. Federici, A coupling approach to modeling heat transfer during a full transient flight cycle. International Journal of Heat and Mass Transfer, 587-605, 110, April 2017.

[31] SK. Godunov, V.S. Ryabenkii, The theory of difference schemes. An introduction, NorthHolland: Amsterdam, (1964).

[32] H-O. Kreiss, Stability theory for difference approximations of mixed initial boundary value problems. I., Math. Comp., 22 (1968) 703-714.

[33] S. Osher, Systems of difference equations with general homogeneous boundary conditions. Trans. Amer. Math. Soc. 137 (1969) 177-201.

[34] B. Gustaffson, H-O. Kreiss, A. Sundström, Stability theory of difference approximations for mixed initial boundary value problems, Math. Comput., 26, 649-686, 1972.

[35] B. Gustafsson, The Godunov-Ryabenkii condition: the beginning of a new stability theory. Technical report 1999-014. Department of Information Technology (1999).

[36] R. El Khoury, M. Errera, K. El Khoury, M. Nemer, Efficiency of coupling schemes for the treatment of steady state fluid-structure thermal interactions. International Journal of Thermal Sciences, 115, 225-235, 2017.

[37] M.-P. Errera, R. Moretti, R. Salem, Y. Bachelier, T. Arrivé, M. Nguyen, A single stable scheme for steady conjugate heat transfer problems, Journal of Computational Physics, 394, 491$502,2019$. 
[38] R. Salem, M. Errera, J. Marty, Adaptive diffusive time-step in conjugate heat transfer interface conditions for thermal-barrier-coated applications, International Journal of Thermal Sciences, 145, 106048, 2019.

[39] G. Gimenez, M.P. Errera, D. Baillis, Y. Smith, F. Pardo, A coupling numerical methodology for weakly transient conjugate heat transfer problems, International Journal of Heat and Mass Transfer 97, 975-989, 2016.

[40] http://elsa.onera.fr

[41] L. Cambier, M. Gazaix, "elsA : an efficient object-oriented solution to CFD complexity", AIAA Aerospace Science Meeting and Exhibit, AIAA 2002-0108, 2002. https://doi.org/10.2514/6.2002-108

[42] L. Cambier, M. Gazaix, S. Heib, S. Plot, M. Poinot, J.-P. Veuillot, J.-F. Boussuge, M. Montagnac, An Overview of the Multi-Purpose elsA Flow Solver, Aerospace Lab, Issue 2, 2011

[43] L. Cambier, S. Heib, S. Plot, The Onera elsA CFD software: input from research and feedback from industry, Mech. \& Ind. 14 (2013) 159-174. doi:10.1051/meca/2013056

[44] http://www.zset-software.com

[45]L. Tessé, J-M Lamet, Radiative Transfer Modeling Developed at Onera for Numerical Simulations of Reactive Flows. Aerospace Lab Journal, Issue 2, March 2011. http://www.aerospacelab-journal.org/al2

[46]L. Tessé, F. Dupoirieux, J. Taine, Monte Carlo modeling of radiative transfer in a turbulent sooty flame, International Journal of Heat and Mass Transfer, Vol. 47, pp. 555-572, 2004. https://doi.org/10.1016/j.ijheatmasstransfer.2003.06.003

[47] O. Rouzaud, L. Tessé, T. Soubrié, A. Soufiani, Ph. Rivière, D. Zeitoun, Influence of radiative heating on a Martian orbiter, Journal of Thermophysics and Heat Transfer, Vol. 22, No. 1, pp. 10-19, January-March 2008. https://doi.org/10.2514/1.28259

[48]L. Tessé, F. Dupoirieux, B. Zamuner, J. Taine, Radiative Transfer in Real Gases Using Reciprocal and Forward Monte Carlo Methods and a Correlated-k Approach. International Journal of Heat and Mass Transfer, Vol. 45, pp. 2797-2814, 2002. https://doi.org/10.1016/S0017-9310(02)00009-1

[49]F. Dupoirieux, L. Tessé, A. Avila, J. Taine, An Optimized Reciprocity Monte Carlo Method for the Calculation of Radiative Transfer in Media of Various Optical Thicknesses. International Journal of Heat and Mass Transfer, Vol. 49, pp. 1310-1319, 2006. https://doi.org/10.1016/j.ijheatmasstransfer.2005.10.009

[50] http://sites.onera.fr/cwipi

[51] A. Jameson, W. Schmidt, E. Turkel, Numerical solutions of the Euler equations by finite volume methods using Runge Kutta time stepping schemes. AIAA Paper 81-1259, 1981.

[52] P. R. Spalart and S. R Allmaras, A One-Equation Turbulence Model for Aerodynamic Flows, La Recherche Aérospatiale, $N^{\circ} 1,5-21,1994$. 